\title{
The genomic basis of circadian and circalunar timing adaptations in a midge
}

Tobias S. Kaiser ${ }^{1,2,3}+$, Birgit Poehn ${ }^{1,3}$, David Szkiba ${ }^{2}$, Marco Preussner $^{4}$, Fritz J. Sedlazeck ${ }^{2} \dagger$, Alexander Zrim² ${ }^{2}$ Tobias Neumann ${ }^{1,2}$, Lam-Tung Nguyen ${ }^{2,5}$, Andrea J. Betancourt ${ }^{6}$, Thomas Hummel ${ }^{3,7}$, Heiko Vogel ${ }^{8}$, Silke Dorner ${ }^{1}$, Florian Heyd ${ }^{4}$, Arndt von Haeseler ${ }^{2,3,5}$ \& Kristin Tessmar-Raible ${ }^{1,3}$

Organisms use endogenous clocks to anticipate regular environmental cycles, such as days and tides. Natural variants resulting in differently timed behaviour or physiology, known as chronotypes in humans, have not been well characterized at the molecular level. We sequenced the genome of Clunio marinus, a marine midge whose reproduction is timed by circadian and circalunar clocks. Midges from different locations show strain-specific genetic timing adaptations. We examined genetic variation in five $C$. marinus strains from different locations and mapped quantitative trait loci for circalunar and circadian chronotypes. The region most strongly associated with circadian chronotypes generates strain-specific differences in the abundance of calcium/calmodulin-dependent kinase II.1 (CaMKII.1) splice variants. As equivalent variants were shown to alter CaMKII activity in Drosophila melanogaster, and C. marinus (Cma)-CaMKII.1 increases the transcriptional activity of the dimer of the circadian proteins Cma-CLOCK and Cma-CYCLE, we suggest that modulation of alternative splicing is a mechanism for natural adaptation in circadian timing.

Around the new or full moon, during a few specific hours surrounding low tide, millions of non-biting midges of the species C. marinus emerge from the sea to perform their nuptial dance. Adults live for only a few hours, during which they mate and oviposit. They must therefore emerge synchronously and-given that embryonic, larval and pupal development take place in the sea-at a time when the most extreme tides reliably expose the larval habitat. The lowest low tides occur predictably during specific days of the lunar month at a specific time of day. Consequently, adult emergence in C. marinus is under the control of circalunar and circadian clocks ${ }^{1,2}$. Notably, although the lowest low tides recur invariably at a given location, their timing differs between geographic locations ${ }^{3}$. Consequently, C. marinus strains from different locations (Extended Data Fig. 1a) show local adaptation in circadian and circalunar emergence times (Extended Data Fig. 1b, c). Crosses between the Jean and Por strains showed that the differences in circadian and circalunar timing are genetically determined ${ }^{4,5}$ and largely explained by two circadian and two circalunar quantitative trait loci (QTLs) ${ }^{6}$.

Studies on timing variation or chronotypes in animals and humans have often focused on candidate genes from the circadian transcriptiontranslational oscillator. In D. melanogaster, polymorphisms in the core circadian clock genes period, timeless and cryptochrome are associated with adaptive differences in temperature compensation ${ }^{7}$, photo-responsiveness of the circadian clock $^{8}$ and emergence rhythms ${ }^{9}$. While these studies offer insights into the evolution of known circadianclock molecules, genome-wide association studies ${ }^{10,11}$ and other forward genetic approaches (reviewed in ref. 12) are essential to provide a comprehensive, unbiased assessment of natural timing variation, for instance underlying human sleep-phase disorders. While the adaptive nature of human chronotypes remains unclear, the chronotypes of $C$. marinus represent evolutionary adaptations to their habitat.
Our study aimed to identify the genetic basis of $C$. marinus adaptation to its specific ecological 'timing niche'. In addition, the genetic dissection of adaptive natural variants of non-circadian rhythms ${ }^{13}$, as also present in C. marinus, may provide an entry point into their unknown molecular mechanisms.

As a starting point for these analyses, we sequenced, assembled, mapped and annotated a C. marinus reference genome.

\section{The Clunio genome and QTLs for timing}

Our reference genome CLUMA_1.0 of the Jean laboratory strain contained $85.6 \mathrm{Mb}$ of sequence (Table 1), close to the previous flowcytometry-based estimate of $95 \mathrm{Mb}^{6}$, underlining that chironomids generally have small genomes ${ }^{14-16}$. The final assembly has a scaffold $\mathrm{N} 50$ of $1.9 \mathrm{Mb}$. Genome-wide genotyping of a mapping family with restriction-site associated DNA sequencing allowed $92 \%$ of the reference sequence to be consistently anchored along a genetic linkage map (Fig. 1a and Extended Data Fig. 2), improving the original linkage map (Supplementary Methods 5). Automated genome annotation resulted in 21,672 gene models. Protein similarity and available transcripts support 14,041 gene models (Supplementary Table 1), within the range of gene counts for D. melanogaster $(15,507)$ and Anopheles gambiae $(13,460)$. Thus, the very small C. marinus genome appears to be complete (Table 1, Extended Data Fig. 3, Supplementary Note 1 and Supplementary Table 2). The C. marinus reference genome makes chironomids the third dipteran subfamily with an annotated genome reconstructed to chromosome scale (Fig. 1a and Extended Data Figs 2, 3b-f).

We performed a basic genome characterization and comparison to other dipterans. We delineated the five C. marinus chromosome arms (Supplementary Note 2, Extended Data Fig. 3c and Supplementary Table 3) and homologized them to D. melanogaster and A. gambiae by synteny comparisons (Extended Data Figs 3 and 4, Supplementary Note 2 and

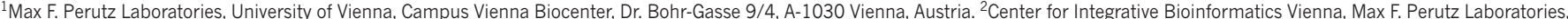

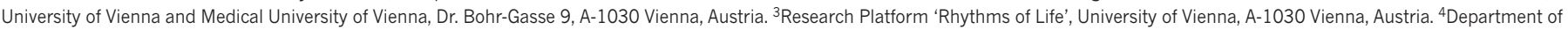

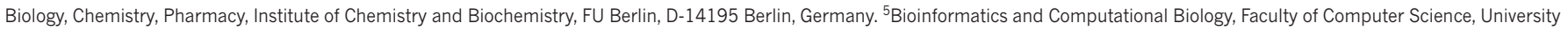
of Vienna, A-1030 Vienna, Austria. ${ }^{6}$ Institute of Population Genetics, Department of Biomedical Sciences, University of Veterinary Medicine Vienna, Josef-Baumann-Gasse 1, A-1210 Vienna, Austria. ${ }^{7}$ Department of Neurobiology, Faculty of Life Sciences, University of Vienna, A-1090 Vienna, Austria. ${ }^{8}$ Department of Entomology, Max Planck Institute for Chemical Ecology, Hans-KnöllStraße 8, D-07745 Jena, Germany. †Present addresses: Max Planck Institute for Evolutionary Biology, August-Thienemann-Straße 2, D-24306 Plön, Germany (T.S.K.); Department of Computer Science, Johns Hopkins University, Baltimore, Maryland 21211, USA (F.J.S.). 
Table 1 | Comparison of the $C$. marinus genome assembly with published model insect genomes

\begin{tabular}{|c|c|c|c|c|}
\hline & $\begin{array}{l}\text { Clunio } \\
\text { marinus }\end{array}$ & $\begin{array}{c}\text { Danaus } \\
\text { plexippus }^{43}\end{array}$ & $\begin{array}{c}\text { Tribolium } \\
\text { castaneum }\end{array}$ & $\begin{array}{c}\text { Apis } \\
\text { mellifera }\end{array}$ \\
\hline Total bases (Mb) & 86 & 278 & 160 & 236 \\
\hline Mapped sequence (\%) & 92 & NA & 90 & 79 \\
\hline Scaffold N50 (Mb) & 1.9 & 0.2 & 1 & 0.4 \\
\hline Contig N50 (kb) & 79 & 50 & 41 & 41 \\
\hline AT content (\%) & 68.3 & 68.4 & 67 & 66 \\
\hline Completeness (\%) & 98.0 & 98.5 & NA & NA \\
\hline Platform & Illumina & $\begin{array}{l}\text { Illumina } \\
+454\end{array}$ & $\begin{array}{l}\text { Sanger } \\
+ \text { BAC }\end{array}$ & $\begin{array}{l}\text { Sanger } \\
+B A C\end{array}$ \\
\hline
\end{tabular}

Supplementary Table 3). We also found the ZW-like sex-linked locus in C. marinus ${ }^{6}$ outside the X chromosome homologue (Supplementary Note 2) and detected an elevated rate of chromosomal re-arrangement (Fig. 1a, Supplementary Note 3 and Extended Data Figs 2, 3b-f, 4). Taken together, the C. marinus reference genome appears well assembled.

As the next step towards identifying the molecular basis of circadian and circalunar timing adaptations in $C$. marinus, we refined the previously identified timing QTL positions ${ }^{6}$ based on the new high-density, restriction-site-associated DNA sequencing markers (Supplementary Table 4 and Supplementary Note 4) and determined the reference sequence corresponding to the QTL confidence intervals (Fig. 1, orange and cyan bars; and Supplementary Table 4). None of the core circadian clock genes were found to be located within these QTLs (Fig. 1a). Only timeout/timeless2, a timeless homologue with a minor role in circadian clock resetting $^{17}$, is located within the QTLs.

\section{Genetic variation in Clunio timing strains}

We then re-sequenced the Por and Jean strains (Extended Data Fig. 1), for which the initial QTL analysis was performed ${ }^{6}$. Two pools of 300 field-caught individuals were sequenced at $>240 \times$ coverage (Supplementary Table 5). Mapping reads against the reference genome identified 1,010,052 single nucleotide polymorphisms (SNPs), 72\% of which were present in both the Por and Jean strains. Based on all SNPs we determined genetic differentiation $\left(F_{\mathrm{ST}}\right)$, genetic diversity $(\theta)$ and short-range linkage disequilibrium (measured as $r^{2}$ ) (Fig. $1 \mathrm{~b}$ and Extended Data Figs 3c, 5a, b).

Genome-wide genetic differentiation between the Por and Jean strains is moderate $\left(F_{\mathrm{ST}}=0.11\right)$, providing a good basis for screening the genome for local timing adaptation based on genetic divergence. According to QTL analysis, the two circadian QTLs explain 85\% of the daily timing difference and the two circalunar QTLs explain the entire monthly timing difference (Supplementary Table 4 and ref. 6). As each locus therefore has a strong effect on timing, selection against maladapted alleles must be strong and timing loci should be strongly differentiated.

Within the confidence intervals of the QTLs, 158 SNPs and 106 indels (insertions or deletions) are strongly differentiated $\left(F_{\mathrm{ST}} \geq 0.8\right.$; Fig. $1 b$ and Extended Data Fig. 5; SNPs, red dots in $F_{\text {ST }}$ panels, for genome-wide comparison see Supplementary Note 5). We compiled a list of candidate genes for circadian and circalunar timing adaptations based on their proximity to differentiated SNPs and indels in the QTLs (Supplementary Table 6). The candidate genes neither comprise core circadian clock genes (timeless $2 /$ timeout, $\max . F_{\mathrm{ST}} \leq 0.5$; average $F_{\mathrm{ST}}=0.07$ ), nor are enriched for any particular pathway (gene ontologyterm analysis; Supplementary Table 7).

\section{Timing phenotype with genotype correlation}

Assuming that the alleles associated with timing adaptation probably originated from standing genetic variation (Supplementary Note 5), genetic variation at timing loci should not vary freely between strains, but rather strains with similar timing should share functionally relevant alleles. To identify such loci, we extended the genomic screen to three additional strains: from Vigo (Vigo), Helgoland (He) and Bergen (Ber; Extended Data Fig. 1 and Supplementary Tables 5, 8). We then tested all five sequenced strains for correlations between genetic differentiation $\left(F_{\mathrm{ST}}\right)$ and timing differences, or geographic distances as a null model (Supplementary Table 8).

Overall, genome-wide genetic differentiation was not correlated with circadian $(r=0.10, P=0.31)$ or circalunar $(r=0.56, P=0.12)$ timing differences, but with geographic distance ('isolation by distance'; $r=0.88, P=0.008$ ). Against this genomic background signal of isolation by distance, we screened the genome in 5-kb sliding windows for peaks of correlation between genetic differentiation and timing, resulting in a correlation score (Fig. $1 \mathrm{~b}$ and Extended Data Fig. 5a, b, CS panels, score ranging from 0 to 5; for details see Methods). Combining the evidence from the Por versus Jean strain $F_{\mathrm{ST}}$ screen (Supplementary Table 6) with these patterns of correlation between timing and genetic divergence reduced the candidate gene list to 49 genes (Supplementary Table 9).

Of particular note, a single region in circadian QTL C2 was strikingly differentiated (Fig. 1b). In this region, linkage disequilbrium in the Por strain was significantly elevated (permutation test; $P=0.002$ ), and genetic diversity significantly decreased in some stretches (permutation test; $P=0.037$ and 0.020 ), compared to the Por genome average. This may indicate a recent episode of selection in Por, potentially during timing adaptation, as this region is also strongly enriched for timingcorrelated polymorphisms (Fig. 1b, CS panel). The most extreme values of genetic differentiation, genetic diversity and timing correlation localize to the CaMKII. 1 locus and the anterior section of a gene homologous to the big bang $(b b g)$ gene.

\section{CaMKII affects the circadian core clock}

Not only does the CaMKII.1 locus harbour the highest number of differentiated polymorphisms (Supplementary Table 9), but CaMKII has also been shown to affect circadian timing. Mouse CaMKII $\alpha$ phosphorylates CLOCK and facilitates its dimerization with BMAL in vivo ${ }^{18}$. Mice with inactive, kinase-dead CaMKII $\alpha^{\mathrm{K} 42 \mathrm{R}}$ have dampened circadian rhythms and a lengthened circadian free-running period $^{18}$. CaMKII also phosphorylates the CLOCK protein ${ }^{19}$ in the D. melanogaster S2 cell line, and in vivo inhibition of Dme-CaMKII in a sensitized background with reduced $\mathrm{Ca}^{2+}$ levels lengthens the circadian free-running period ${ }^{20}$, suggesting that the role of CAMKII in circadian timing is conserved across animals.

To determine whether CaMKII can also affect the circadian core clock in C. marinus, we tested the effect of $\mathrm{Cma}$-CaMKII. 1 in a cell-based assay using D. melanogaster S2 cells ${ }^{19,21}$. We repeated previous experiments ${ }^{19}$ showing that the chemical inhibition of endogenous Dme-CaMKII reduces the amount of generated luciferase (Extended Data Fig. 6a), whereas addition of a $\left[\mathrm{Ca}^{2+}\right]$-independent, and therefore constitutively active, variant of CaMKII (mouse, T286D) increases luciferase amounts (Extended Data Fig. 6b). Then we generated constructs for C. marinus clock, C. marinus cycle, and mutated kinase-dead (K42R) and $\left[\mathrm{Ca}^{2+}\right]-$ independent (T286D) versions of Cma-CaMKII.1. Transfection of Cmaclock and Cma-cycle into D. melanogaster S2 cells leads to luciferase activity driven from the $3 \mathrm{X} 69$ promoter derived from the Dme-period promoter (Fig. 2a). The addition of $\left[\mathrm{Ca}^{2+}\right]$-independent $\mathrm{Cma}$ CaMKII. $1^{\mathrm{T} 286 \mathrm{D}}$ leads to a substantial increase in the luciferase signal (Fig. 2a), whereas addition of the kinase-dead Cma-CaMKII. $1^{\mathrm{K} 42 \mathrm{R}}$ does not enhance luciferase activity (Fig. 2a). These data suggest that CaMKII kinase activity enhances E-box dependent transcription, as indicated by luciferase production driven by the 3 X69promoter, via the CLOCKCYCLE dimer in C. marinus.

\section{CaMKII.1 splicing correlates with timing}

We then investigated how polymorphisms in the Cma-CaMKII.1 locus affect the enzyme. We found two CaMKII.1 alleles: one in the early 
a

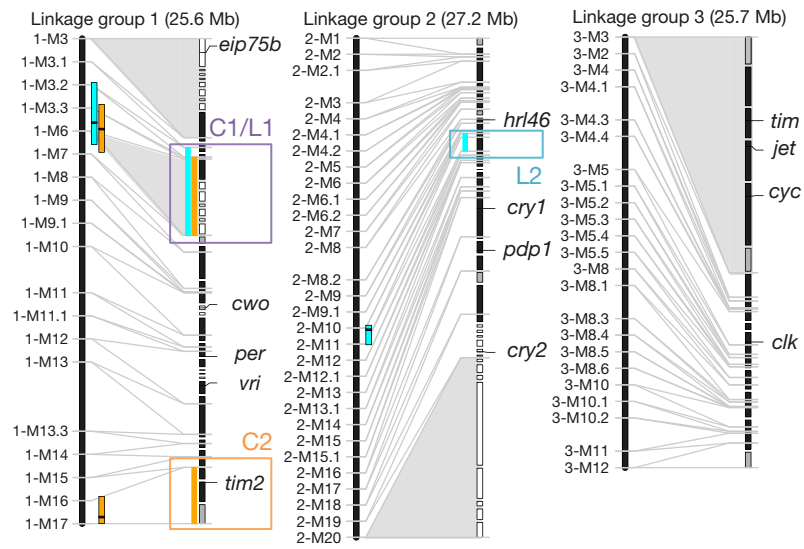

b

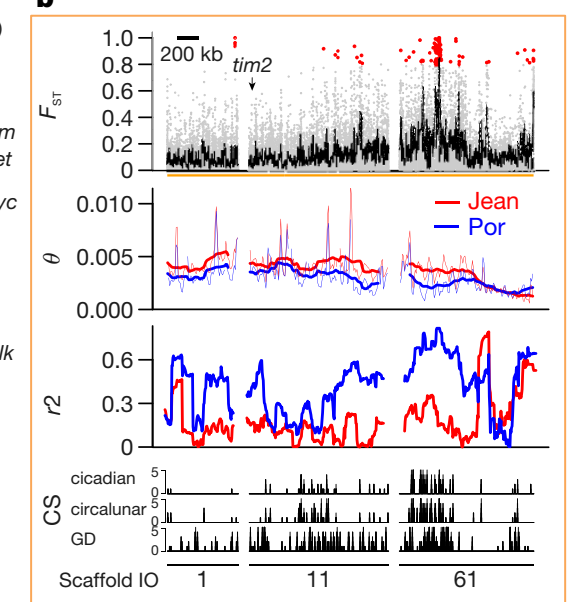

Por and Jean strains (in blue and red, respectively, in middle two panels). Top panel, genetic differentiation for single SNPs (red dots) and in $5-\mathrm{kb}$ windows (black line). Second panel, genetic diversity $(\theta)$ in $20-\mathrm{kb}$ (thin line) and $200-\mathrm{kb}$ (thick line) windows. Third panel, linkage disequilibrium $\left(r^{2}\right)$ in 100-kb windows. Bottom panel, correlation score (CS) for genetic differentiation with values for circadian timing (top), circalunar timing (middle) and geographic distance (bottom) for Vigo, Jean, Por, He and Ber strains. Bottom numbers, scaffold IDs. For further details, including QTLs C1/L1 and L2, see Extended Data Fig. 5a, b. emerging Por, He and Ber strains, and another in the late emerging Jean and Vigo strains. Most strain-specific polymorphisms are located in introns (Fig. 2b, c and Supplementary Table 9). If these polymorphisms were meaningful, then they should affect CaMKII.1 expression and/or splicing. Cma-CaMKII.1 has four functional domains ${ }^{22}$ (Fig. 2b). The majority of differentiated polymorphisms cluster in the region of the variable linker domain (Fig. 2b, c), including a 125-bp insertion (red dot in Fig. 2c; Extended Data Fig. 7). We identified four alternatively spliced full-length transcripts of Cma-CaMKII.1 (RA-RD), which differ in the length of the linker (Fig. 2b). High-coverage RNA sequencing gave evidence for differential exon usage between the Jean and Por strains, as well as for previously non-annotated exons within the variable linker region (Extended Data Fig. 6c). PCR and Sanger sequencing confirmed several partial transcripts of additional splice variants of the linker region (RE-RO; Fig. 2b). We used transcript-specific qPCR to quantify all transcripts from third instar larvae. Generally, transcripts $\mathrm{RE}-\mathrm{RO}$ are expressed at very low levels. Of those, only RO showed quantifiable expression differences between the Jean and Por strains (Fig. 3a and Extended Data Fig. 6d). Importantly, transcript-specific qPCR confirmed significant differential expression of the major transcripts in the Jean versus Por strains (Fig. 3a, Extended Data Fig. 6d), matching the RNA-sequencing (RNA-seq) data (Extended Data Fig. 6c). Consistently, variants with long linkers (RA, RB) showed higher expression in the Por strain, whereas shorter variants $(\mathrm{RD}, \mathrm{RO})$ showed higher expression in the Jean strain (Fig. 3a and Extended Data Fig. 6c, d).

If the detected differences in the abundance of CaMKII.1 splice variants are associated with the timing differences, they should be directly caused by the strain-specific polymorphisms at the CaMKII. 1 locus. In order to test this, we generated minigenes that contained the alternatively spliced linker region of the CaMKII.1 locus from either the Jean or Por strains. The two minigenes were transfected into cells of the D. melanogaster $\mathrm{S} 2 \mathrm{R}+$ cell line and expression of splice variants was analysed by radioactive RT-PCR (Fig. 3b, c). We detected four variants, corresponding to splice variants $\mathrm{RB}, \mathrm{RC}, \mathrm{RD}$ and $\mathrm{RO}$. All variants

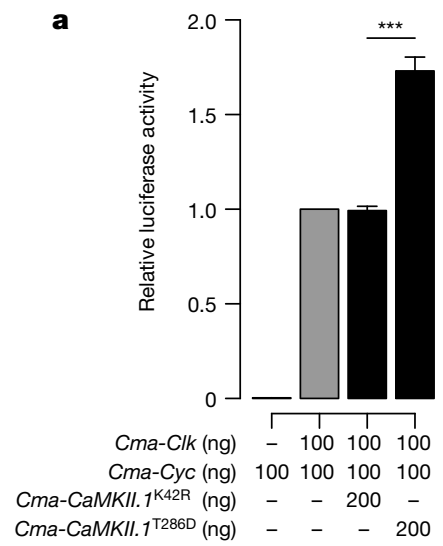

Figure 2 | CaMKII.1 regulates Clk and Cyc transcriptional activity and exhibits strain specific splice variants. a, Additional C. marinus CaMKII.1 increases the transcriptional activity of C. marinus Clk and Cyc in a D. melanogaster S2 cell luciferase assay using the 3X69 E-box containing enhancer (period 3X69-luc (ref. 21)). Data are represented as mean \pm s.e.m.; two-sided Welch two-sample $t$-test; biological replicates,

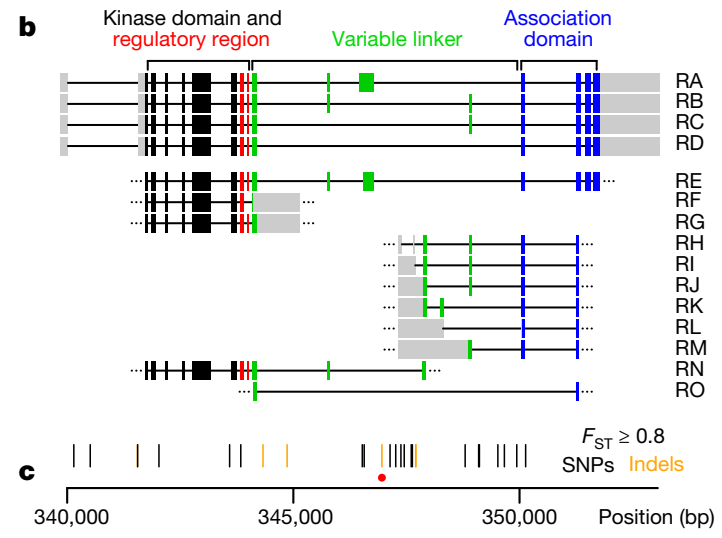

$n=5$, except for no $c l k$ control, $n=3$, each biological replicate represents the average of three preparation replicates. $* * * P<0.0005$. b. Exons of full (RA-RD) and partial (RE-RO) Cma-CaMKII.1 transcripts. c, Distribution of SNPs (black), indels (orange) and a 125-bp insertion (red dot) along the Cma-CaMKII. 1 locus, all with $F_{\mathrm{ST}} \geq 0.8$. 

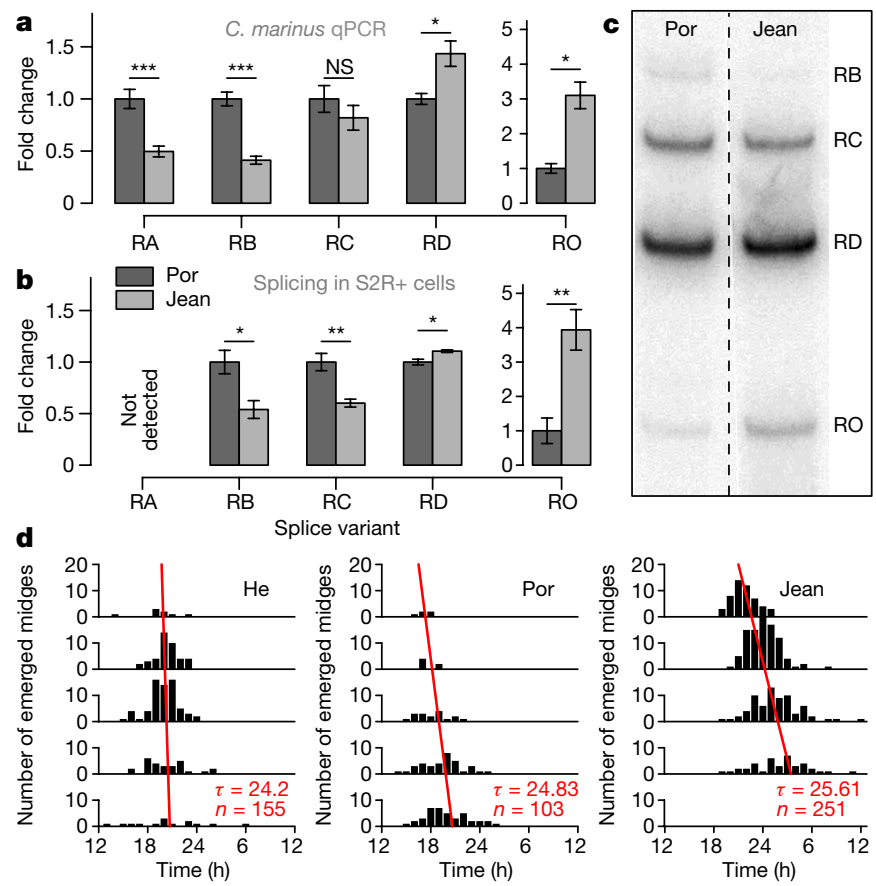

Figure 3 Differential CaMKII.1 splicing depends on sequence differences in the CaMKII.1 locus and correlates with endogenous circadian period lengths. a, qPCR values for CaMKII.1 splice variants from Por and Jean strains, normalized to Por (for non-normalized data, see Extended Data Fig. 6d). Data are represented as mean \pm s.e.m.; Por, $n=9$ biological replicates; Jean, $n=10$; RO, Por, $n=3$; Jean $n=8$; RO was not detected in six Por biological replicates, suggesting an even larger expression difference; two-sided Wilcoxon rank-sum test; ${ }^{*} P<0.05$; $* * P<0.005$; $* * * P<0.0005$; NS, not significant; Holm correction for multiple testing. For RNA-seq data quantification see Extended Data Fig. 6c. b, Differential splicing of the CaMKII. 1 linker region in D. melanogaster S2R + cells, normalized to Por, $n=7$ biological replicates; two-sided two-sample $t$-test, otherwise as a. c, Representative phosphorimaging gel sections as quantified for $\mathbf{b}$, two separate lanes from the same gel (for full gel, see Source Data). d, Free-running rhythm of adult emergence under constant dim white light (approximately $100 \mathrm{~lx}$ ). He and Por share CaMKII.1 alleles, while Jean has the other allele. To calculate the free-running period, time between subsequent emergence peaks was averaged, weighting each peak by the number of individuals.

showed the same strain-specific abundance differences in the S2R+ cell assay as in the C. marinus strains in vivo (Fig. 3a, b). As the cellular context is the same for both the Jean and Por minigenes in the S2R+ assay, trans-acting elements can be excluded as the cause of differential splicing, implying that it is a direct result of the genomic sequence differences at the Cma-CaMKII.1 locus. While splice variants RB, RC and $\mathrm{RD}$ and their constituting exons are conserved in D. melanogaster (see Flybase annotations and ref. 23), a D. melanogaster RA counterpart does not exist. This may explain why this variant is undetectable in $\mathrm{S} 2 \mathrm{R}+$ cells (Fig. $3 \mathrm{~b})$.

\section{From splice variants to timing differences}

CaMKII linker-length variants have been investigated in several species. D. melanogaster CaMKII isoforms corresponding to the RB, $\mathrm{RC}$ and $\mathrm{RD}$ variants of $C$. marinus have different substrate affinities and rates of target phosphorylation ${ }^{23}$. These activity differences are explained by the fact that CaMKII functions as a dodecamer, and the linker length determines the compactness and thus the substrate accessibility of the holoenzyme-enzymes with long linkers have higher activity. This structure-function relationship is possibly universal, as it is conserved between humans and C. elegans ${ }^{22,24}$.

Inactivation or inhibition of CaMKII lengthens circadian periods in mouse and fruitflies ${ }^{18,20}$. A connection between circadian period length and phase of activity in light-dark cycles is known from mutations in period in D. melanogaster ${ }^{25}$ and human chronotypes ${ }^{26}$. These findings imply that in C. marinus the more active and more readily $\mathrm{Ca}^{2+}$-activated, long-linker CaMKII.1 variants should advance adult emergence by shortening the circadian clock period. Indeed, we find that the early emerging Por and He strains, which possess the same long-linker biased CaMKII.1 alleles, have shorter free-running circadian clock periods than the late emerging Jean strain (Fig. 3d).

Integrating our results with those from the aforementioned literature, we propose that regulation of the ratio of CaMKII.1 splice variants constitutes an evolutionary mechanism to adapt circadian timing (Extended Data Fig. 8): differences in the genomic sequence of CaMKII.1 lead to differential CaMKII.1 splicing and activity. Among a number of possible targets, this influences CLOCK-CYCLE dimerdependent transcription, which in turn affects circadian period length and ultimately results in differences in adult emergence time.

\section{Discussion}

Annual, lunar and tidal rhythms, as well as natural timing variation between individuals, are important and widespread phenomena that are poorly understood. The $C$. marinus reference genome and the genetic variation panel for five strains with differing circadian and circalunar timing establish new resources for further studies of these topics.

We identified C. marinus orthologues for all core circadian clock genes, none of which appear to be involved in circadian or circalunar timing adaptations. For circalunar timing, this supports the molecular independence of the circalunar clock from the circadian clock, as reported for Platynereis dumerilii ${ }^{27}$.

For circadian timing, strain-specific modulation in alternative splicing of CaMKII.1 emerges as a possible mechanism for natural adaptation. In the light of previous experiments in D. melanogaster and mice $^{18-20,23}$, it seems most likely that differences in CaMKII activity of the different splice forms lead to circadian timing differences via phosphorylation of CLOCK-CYCLE (Extended Data Fig. 8).

It is also conceivable that CaMKII affects circadian timing via other targets. For example, CaMKII is known to phosphorylate the cAMP response element binding protein (CREB) ${ }^{28,29}$. CREB is linked to the circadian clock by cAMP response elements (CRE) in the promoters of the period and timeless genes ${ }^{30,31}$, and by physical interaction of the CREB-binding protein (CBP) with CREB, CLOCK and CYCLE ${ }^{32,33}$. Furthermore, one of the most well-studied roles of CaMKII is the morphological modulation of neuronal plasticity and connectivity ${ }^{34-36}$. Such changes in connectivity have been increasingly implicated as part of the circadian timing mechanism in D. melanogaster and mammals ${ }^{37}$. Interestingly, the role of CaMKII in shaping neuronal connectivity has also been suggested to link to several neuropsychiatric diseases ${ }^{38}$, which often co-occur with chronobiological disruption $\mathrm{s}^{39-42}$. Further studies are needed to determine whether the modulation of CaMKII activity constitutes a molecular link between these phenomena.

Online Content Methods, along with any additional Extended Data display items and Source Data, are available in the online version of the paper; references unique to these sections appear only in the online paper.

Received 17 March 2015; accepted 10 October 2016.

Published online 21 November 2016.

1. Neumann, D. Die lunare und tägliche Schlüpfperiodik der Mücke Clunio. Steuerung und Abstimmung auf die Gezeitenperiodik. Z. Vgl. Physiol. 53, 1-61 (1966).

2. Neumann, D. Temperature compensation of circasemilunar timing in the intertidal insect Clunio. J. Comp. Physiol. 163, 671-676 (1988).

3. ADMIRALTY Tide Tables (UKHO, 2014).

4. Neumann, D. Genetic adaptation in emergence time of Clunio populations to different tidal conditions. Helgoländer Wiss. Meeresunters. 15, 163-171 (1967).

5. Kaiser, T. S., Neumann, D. \& Heckel, D. G. Timing the tides: genetic control of diurnal and lunar emergence times is correlated in the marine midge Clunio marinus. BMC Genet. 12, 49 (2011). 
6. Kaiser, T. S. \& Heckel, D. G. Genetic architecture of local adaptation in lunar and diurnal emergence times of the marine midge Clunio marinus (Chironomidae, Diptera). PLoS One 7, e32092 (2012).

7. Sawyer, L. A. et al. Natural variation in a Drosophila clock gene and temperature compensation. Science 278, 2117-2120 (1997).

8. Sandrelli, F. et al. A molecular basis for natural selection at the timeless locus in Drosophila melanogaster. Science 316, 1898-1900 (2007)

9. Pegoraro, M. et al. Molecular evolution of a pervasive natural amino-acid substitution in Drosophila cryptochrome. PLoS One 9, e86483 (2014)

10. Lane, J. M. et al. Genome-wide association analysis identifies novel loci for chronotype in 100,420 individuals from the UK Biobank. Nat. Commun. 7, 10889 (2016).

11. Hu, Y. et al. GWAS of 89,283 individuals identifies genetic variants associated with self-reporting of being a morning person. Nat. Commun. 7, 10448 (2016).

12. Jones, C. R., Huang, A. L., Ptáček, L. J. \& Fu, Y.-H. Genetic basis of human circadian rhythm disorders. Exp. Neurol. 243, 28-33 (2013).

13. Tessmar-Raible, K., Raible, F. \& Arboleda, E. Another place, another timer: marine species and the rhythms of life. BioEssays 33, 165-172 (2011)

14. Gusev, O. et al. Comparative genome sequencing reveals genomic signature of extreme desiccation tolerance in the anhydrobiotic midge. Nat. Commun. $\mathbf{5}$, 4784 (2014).

15. Cornette, R. et al. Chironomid midges (Diptera, chironomidae) show extremely small genome sizes. Zoolog. Sci. 32, 248-254 (2015).

16. Kelley, J. L. et al. Compact genome of the Antarctic midge is likely an adaptation to an extreme environment. Nat. Commun. 5, 4611 (2014)

17. Benna, C. et al. Drosophila timeless 2 is required for chromosome stability and circadian photoreception. Curr. Biol. 20, 346-352 (2010).

18. Kon, N. et al. CaMKII is essential for the cellular clock and coupling between morning and evening behavioral rhythms. Genes Dev. 28, 1101-1110 (2014).

19. Weber, F., Hung, H. C., Maurer, C. \& Kay, S. A. Second messenger and Ras/ MAPK signalling pathways regulate CLOCK/CYCLE-dependent transcription. J. Neurochem. 98, 248-257 (2006)

20. Harrisingh, M. C., Wu, Y., Lnenicka, G. A. \& Nitabach, M. N. Intracellular Ca2 ${ }^{+}$ regulates free-running circadian clock oscillation in vivo. J. Neurosci. 27, 12489-12499 (2007).

21. Nawathean, P. \& Rosbash, M. The doubletime and CKII kinases collaborate to potentiate Drosophila PER transcriptional repressor activity. Mol. Cell 13, 213-223 (2004).

22. Chao, L. H. et al. A mechanism for tunable autoinhibition in the structure of a human $\mathrm{Ca}_{2}^{+}$/calmodulin-dependent kinase II holoenzyme. Cell 146, 732-745 (2011).

23. GuptaRoy, B. et al. Alternative splicing of Drosophila calcium/calmodulindependent protein kinase II regulates substrate specificity and activation. Brain Res. Mol. Brain Res. 80, 26-34 (2000).

24. Chao, L. H. et al. Intersubunit capture of regulatory segments is a component of cooperative CaMKII activation. Nat. Struct. Mol. Biol. 17, 264-272 (2010).

25. Hamblen-Coyle, M. J., Wheeler, D. A., Rutila, J. E., Rosbash, M. \& Hall, J. C. Behavior of period-altered circadian rhythm mutants of Drosophila in light: dark cycles (Diptera: Drosophilidae). J. Insect Behav, 5, 417-446 (1992)

26. Brown, S. A. et al. Molecular insights into human daily behavior. Proc. Natl Acad. Sci. USA 105, 1602-1607 (2008).

27. Zantke, J. et al. Circadian and circalunar clock interactions in a marine annelid. Cell Rep. 5, 99-113 (2013).

28. Sun, P., Enslen, H., Myung, P. S. \& Maurer, R. A. Differential activation of CREB by $\mathrm{Ca} 2^{+}$/calmodulin-dependent protein kinases type II and type IV involves phosphorylation of a site that negatively regulates activity. Genes Dev. 8, 2527-2539 (1994).

29. Wu, X. \& McMurray, C. T. Calmodulin kinase II attenuation of gene transcription by preventing CAMP response element-binding protein (CREB) dimerization and binding of the CREB-binding protein. J. Biol. Chem. 276, 1735-1741 (2001).

30. Belvin, M. P., Zhou, H. \& Yin, J. C. P. The Drosophila dCREB2 gene affects the circadian clock. Neuron 22, 777-787 (1999).

31. Okada, T. et al. Promoter analysis for daily expression of Drosophila timeless gene. Biochem. Biophys. Res. Commun. 283, 577-582 (2001).

32. Lim, C. et al. Functional role of CREB-binding protein in the circadian clock system of Drosophila melanogaster. Mol. Cell. Biol. 27, 4876-4890 (2007).

33. Lee, Y. et al. Coactivation of the CLOCK-BMAL1 complex by CBP mediates resetting of the circadian clock. J. Cell Sci. 123, 3547-3557 (2010).

34. Kalil, K., Li, L. \& Hutchins, B. I. Signaling mechanisms in cortical axon growth, guidance, and branching. Front. Neuroanat. 5, 62 (2011).

35. Hell, J. W. CaMKII: claiming center stage in postsynaptic function and organization. Neuron 81, 249-265 (2014)
36. McVicker, D. P. Millette, M. M. \& Dent, E. W. Signaling to the microtubule cytoskeleton: an unconventional role for CaMKII. Dev. Neurobiol. 75, 423-434 (2015).

37. Bosler, O., Girardet, C., Franc, J.-L., Becquet, D. \& François-Bellan, A.-M. Structural plasticity of the circadian timing system. An overview from flies to mammals. Front. Neuroendocrinol. 38, 50-64 (2015).

38. Robison, A. J. Emerging role of CaMKII in neuropsychiatric disease. Trends Neurosci. 37, 653-662 (2014).

39. Wulff, K., Gatti, S., Wettstein, J. G. \& Foster, R. G. Sleep and circadian rhythm disruption in psychiatric and neurodegenerative disease. Nat. Rev. Neurosci. 11, 589-599 (2010).

40. Levandovski, R. et al. Depression scores associate with chronotype and social jetlag in a rural population. Chronobiol. Int. 28, 771-778 (2011).

41. Zordan, M. A. \& Sandrelli, F. Circadian clock dysfunction and psychiatric disease: could fruit flies have a say? Front. Neurol. 6, 80 (2015).

42. Logan, R. W. et al. Chronic stress induces brain region-specific alterations of molecular rhythms that correlate with depression-like behavior in mice. Biol. Psychiatry 78, 249-258 (2015).

43. Zhan, S., Merlin, C., Boore, J. L. \& Reppert, S. M. The monarch butterfly genome yields insights into long-distance migration. Cell 147, 1171-1185 (2011)

44. Richards, S. et al. The genome of the model beetle and pest Tribolium castaneum. Nature 452, 949-955 (2008).

45. Weinstock, G. M. et al.; Honeybee Genome Sequencing Consortium. Insights into social insects from the genome of the honeybee Apis mellifera. Nature $\mathbf{4 4 3}$ 931-949 (2006).

Supplementary Information is available in the online version of the paper.

Acknowledgements We thank the members of the Tessmar-Raible, Raible and von Haeseler groups for discussions, and S. Bannister and F. Raible for comments on the manuscript. The research leading to these results has received funding from the research platform 'Rhythms of Life' of the University of Vienna to K.T.-R., T.H. and A.v.H., the FWF (http://www.fwf.ac.at/) START award (\#AY0041321), the HFSP (http://www.hfsp.org/) research grant (\#RGY0082/2010), the ERC (FP7/2007-2013)/ERC Grant Agreement 337011 to K.T.-R. and the DFG grant (\#HE5398/4-1) to F.H. T.S.K. was supported by the Vienna International PostDoctoral Program for Molecular Life Sciences (VIPS), L.-T.N. and A.v.H. by the University of Vienna Initiativkolleg I059-N. The funders had no role in study design, data collection and analysis, decision to publish, or preparation of the manuscript.

Author Contributions T.S.K., K.T.-R. and A.v.H. conceived and designed the study and interpreted the data; T.S.K. carried out field sampling, chronobiological experiments, (super-) scaffolding, genetic/QTL mapping and population genomics; L.-T.N., T.N. and T.S.K. carried out the assembly to contigs; T.S.K. and T.N. carried out the assembly filtering; T.S.K. and F.J.S. carried out gap closing and repeated edge removal; T.S.K., F.J.S. and H.V. carried out RNA-seq; H.V. generated the cDNA library; T.S.K. and D.S. carried out genome annotation; T.S.K. and T.N. carried out analysis of genome completeness; T.S.K. and A.Z. carried out chromosome homology and synteny comparisons; A.J.B. carried out the estimation of linkage disequilibrium; D.S. and T.S.K. carried out the SNP effects and GO term analysis; B.P., T.S.K. and S.D. carried out Cma-CaMKII.1 analyses; M.P. and F.H. carried out the minigene assay; T.H. contributed material; T.S.K. and K.T.-R. wrote the manuscript.

Author Information Reprints and permissions information is available at www.nature.com/reprints. The authors declare no competing financial interests. Readers are welcome to comment on the online version of the paper. Correspondence and requests for materials should be addressed to K.T.-R. (kristin.tessmar@mfpl.ac.at) or T.S.K. (kaiser@evolbio.mpg.de).

Reviewer Information Nature thanks M. Rosbash and the other anonymous reviewer(s) for their contribution to the peer review of this work.

This work is licensed under a Creative Commons Attribution 4.0 International (CC BY 4.0) licence. The images or other third party material in this article are included in the article's Creative Commons licence, unless indicated otherwise in the credit line; if the material is not included under the Creative Commons licence, users will need to obtain permission from the licence holder to reproduce the material. To view a copy of this licence, visit http://creativecommons.org/licenses/by/4.0/. 


\section{METHODS}

No statistical methods were used to predetermine sample size. The experiments were not randomized and the investigators were not blinded to allocation during experiments and outcome assessment.

Animal culture and light regimes. The C. marinus laboratory stocks were bred according to Neumann ${ }^{1}$, care was provided by the MFPL aquatic facility. Briefly, C. marinus were kept in $20 \times 20 \times 5 \mathrm{~cm}$ plastic containers with sand and natural seawater diluted to $15 \%$ with desalted water, fed diatoms (Phaeodactylum tricornutum, strain UTEX 646) in early larval stages and nettle powder in later stages. Temperature in the climate chambers was set to $20^{\circ} \mathrm{C}$ and the light-dark cycle was 12:12 (except where noted differently). Moonlight was simulated with an incandescent flashlight bulb (about $1 \mathrm{~lx}$ ), which was switched on all night for four successive nights every 30 days.

Genome assembly. The genome assembly process (Extended Data Fig. 9a) was based on three sequencing libraries (Supplementary Table 10): a 0.2-kb insert library was prepared from a single adult male of the Jean laboratory strain (established from field samples taken at St. Jean-de-Luz, France, in 2007; >12 generations in the laboratory), which was starved and kept in seawater with penicillin (60 units per $\mathrm{ml})$, streptomycin $\left(60 \mu \mathrm{g} \mathrm{ml}^{-1}\right)$ and neomycin $\left(120 \mu \mathrm{g} \mathrm{ml}^{-1}\right)$ during the last 2 weeks of development. DNA was extracted with a salting-out method ${ }^{46}$, sheared on a Covaris S2 sonicator (frequency sweeping mode; $4^{\circ} \mathrm{C}$; duty cycle, $10 \%$; intensity, 7 ; cycles per burst, 300; microTUBE AFA fibre $6 \times 16 \mathrm{~mm} ; 30 \mathrm{~s}$ ) and prepared for Illumina sequencing with standard protocols. A $2.2-\mathrm{kb}$ and a $7.6-\mathrm{kb}$ insert library were prepared from a polymorphic DNA pool of $>300$ field-caught Jean adult males by Eurofins MWG Operon (Ebersberg, Germany) according to the manufacturer's protocol. Each library was sequenced in one lane of an Illumina HiSeq2000 with 100-bp paired-end reads at the Next Generation Sequencing unit of the Vienna Biocenter Core Facilities (http://vbcf.ac.at).

Reads were filtered for read quality, adaptor and spacer sequences with cutadapt $^{47}(-\mathrm{b}-\mathrm{n} 3-\mathrm{e} 0.1-\mathrm{O} 8-\mathrm{q} 20-\mathrm{m} 13)$ and duplicates were removed with fastq-mcf from ea-utils ${ }^{48}(-\mathrm{D} 70)$. Read pairs were interleaved with ngm-utils ${ }^{49}$, leaving only paired reads. Contamination with human DNA found in the $0.2-\mathrm{kb}$ library was removed by deleting reads matching the human genome at a phred-scaled quality score $\geq 20$ (alignment with $\mathrm{BWA}^{50}$ ).

Assembly into contigs with Velvet ${ }^{51}$ (scaffolding disabled; 57-bp kmers as determined by VelvetOptimiser ${ }^{52}$ ) was based solely on the less polymorphic $0.2-\mathrm{kb}$ library. About 600 remaining adaptor sequences at the ends of assembled contigs were trimmed with cutadapt $(-\mathrm{O} 8-\mathrm{e} 0.1-\mathrm{n} 3)$. For assembly statistics see Supplementary Table 11.

Scaffolding of the contigs was based on all three libraries and performed with SSPACE ${ }^{53}$ in two iterations, that is, scaffolds from the first round were scaffolded again. Using different parameters in the iterations (Supplementary Table 12) allowed different connections to be made and thus increased scaffold connectivity (Supplementary Table 13). The effect is probably owing to the polymorphic nature of the 2.2-kb and 7.6-kb libraries; it results in a 'population-consensus most common arrangement of the scaffolds. The iterative scaffolding process was performed with and without applying a size cut-off excluding contigs $<1 \mathrm{~kb}$, resulting in two independent assemblies (CLUMA_0.3 and CLUMA_0.4; see Extended Data Fig. 9a), which differed in overall connectivity and sequence content (Supplementary Table 11), but also in the identity and structure of the large scaffolds. In order to combine both connectivity and sequence content, and in order to resolve the contradictions in the structure of the largest scaffolds, the two assemblies were compared and reconciled in a manual super-scaffolding process, as detailed in Supplementary Method 1. Briefly, the overlap of scaffolds from the two assemblies was tested with BLAST searches and represented in a graphical network structure. Scaffolds with congruent sequence content in both assemblies would result in a linear network, whereas scaffolds with contradictory sequence content would result in branching networks. At the same time, both assemblies were subject to genetic linkage mapping based on genotypes obtained from restriction-site-associated DNA sequencing (RAD sequencing) of a published mapping family ${ }^{6}$ (Supplementary Method 2). The resulting genetic linkage information served to resolve the branching networks into the longest possible unambiguous linear sub-networks with consistent genetic linkage information (see scheme A in Supplementary Method 1). Finally, the structure of the resulting super-scaffolds was coded in YAML format and translated into DNA sequence with Scaffolder ${ }^{54}$, resulting in 75 mapped super-scaffolds.

The remaining small and unmapped scaffolds were filtered for fragments of the mitochondrial genome, the histone gene cluster and 18S/28S ribosomal rDNA gene cluster, which were assembled separately (Supplementary Method 3; Extended Data Fig. 10). Unmapped scaffolds were also filtered for obvious contamination from other species (Supplementary Method 3). The degree to which the remaining unmapped scaffolds are leftover polymorphic variants of parts of the mapped super-scaffolds was estimated by blasting the former against the latter (Supplementary Method 3 and Supplementary Table 14).
All scaffolds were subject to gap closing with GapFiller ${ }^{55}$ and repeated edges, that is, gaps with almost identical sequences at both sides that are generally not closed because of genetic polymorphisms, were assessed and if possible removed with a custom script (Supplementary Method 4; code available supplied as Source Data File).

The final assembly CLUMA_1.0 was submitted under project PRJEB8339 (75 mapped scaffolds; 23,687 unmapped scaffolds $\geq 100 \mathrm{bp}$ ). The assembly and further information can also be obtained from ClunioBase (http://cluniobase.cibiv. univie.ac.at).

Reconstruction of chromosomes and QTL analysis. Genetic linkage information for the final 75 super-scaffolds was obtained by repeating read mapping to genotype calling for the RAD sequencing experiment as described above (Supplementary Method 2), but now with assembly CLUMA_1.0 as a reference. This allowed us to place and orient super-scaffolds along the genetic linkage map (Fig. 1a and Extended Data Fig. 2). The positions of the recombination events within a scaffold were approximated as the middle between the positions of the two RAD markers between which the marker pattern changed from one map location to the next. The published genetic linkage map was refined and revised (Supplementary Method 5 and Extended Data Fig. 2). Based on the refined linkage map, QTL analysis of the published mapping family was repeated as described ${ }^{6}$ (Supplementary Table 4 and Supplementary Note 5). Using the correspondence between the reference assembly and the genetic linkage map, we were able to directly identify the genomic regions corresponding to the confidence intervals of the QTLs (Fig. 1 and Extended Data Fig. 5a, b).

Transcript sequencing. Assembled transcripts of a normalized cDNA library of all life stages and various $C$. marinus strains (454 sequencing) were available from previous experiments and RNA sequencing data was available for Jean strain adults (Illumina sequencing). Furthermore, specifically for genome annotation, RNA from 80 third instar larvae from the Jean and Por laboratory strains each was prepared for RNA sequencing according to standard protocols (Supplementary Method 6). Each sample was sequenced on a single lane of an Illumina HiSeq 2000. All transcript reads were submitted to the European Nucleotide Archive (ENA) under project PRJEB8339.

For the adult and larval RNA sequencing data, raw reads were quality checked with fastqc ${ }^{56}$, trimmed for adaptors quality with cutadapt ${ }^{47}$ and filtered to contain only read pairs using the interleave command in ngm-utils ${ }^{49}$. Reads were assembled separately for larvae and adults with Trinity ${ }^{57}$ (path_reinforcement_distance: 25; maximum paired-end insert size: $1,500 \mathrm{bp}$; otherwise default parameters).

Genome annotation. Automated annotation was performed with MAKER2 ${ }^{58}$ Repeats were masked based on all available databases in repeatmasker. MAKER2 combined evidence from assembled transcripts (see above), mapped protein data sets from Culex quinquefasciatus (CpipJ1), Anopheles gambiae (AgamP3), Drosophila melanogaster (BDGP5), Danaus plexippus (DanPle_1.0), Apis mellifera (Amel4.0), Tribolium castaneum (Tcas3), Strigamia maritima (Smar1) and Daphnia pulex (Dappu1) and ab initio gene predictions with AUGUSTUS ${ }^{59}$ and SNAP 60 into gene models. AUGUSTUS was trained for C. marinus based on assembled transcripts from the normalized cDNA library. SNAP was run with parameters for A. mellifera, which had the highest congruence with known C. marinus genes in preliminary trials (Supplementary Method 7). MAKER was set to infer gene models from all evidence combined (not transcripts only) and gene predictions without transcript evidence were allowed. Splice variant detection was enabled, single-exon genes had to be larger than $250 \mathrm{bp}$ and intron size was limited to a maximum of $10 \mathrm{~kb}$.

All gene models within the QTL confidence intervals, as well as all putative circadian clock genes and light receptor genes were manually curated: exon-intron boundaries were corrected according to transcript evidence (approximately 500 gene models), chimeric gene models were separated into the underlying individual genes (approximately 100 gene models separated into around 300 gene models) and erroneously split gene models were joined (approximately 15 gene models). Finally, this resulted in 21,672 gene models, which were given IDs from CLUMA CG000001 to CLUMA_CG021672 ('CLUMA' for Clunio marinus, following the controlled vocabulary of species from the UniProt Knowledgebase; CG for 'computated gene'). Splice variants of the same gene (detected in 752 gene models) were identified by the suffix ' $-R A$,' '-RB' and so on, and the corresponding proteins by the suffix '-PA,' '-PB' and so forth.

Gene models were considered as supported if they overlapped with mapped transcripts or protein data (Supplementary Table 1). Gene counts for D. melanogaster were retrieved from BDGP5, version 75.546 and for A. gambiae from AgamP3, version 75.3. The putative identities of the C. marinus gene models were determined in reciprocal BLAST searches, first against UniProtKB/ Swiss-Prot (8,379 gene models assigned) and if no hit was found, second against the non-redundant protein sequences (nr database) at NCBI ( 1,802 additional genes assigned). Reciprocal best hits with an $e$ value $<1 \times 10^{-10}$ were considered putative orthologues (termed 'putative gene $X$ '), non-reciprocal hits with the same $e$ value were considered paralogues (termed 'similar to'). All remaining gene models 
were searched against the PFAM database of protein domains (111 gene models assigned; termed 'gene containing domain X'). If still no hit was found, the gene models were left unassigned ('NA').

Synteny comparisons. Genome-wide synteny between the C. marinus, D. melanogaster and A. gambiae genomes was assessed based on reciprocal best BLAST hits $\left(e\right.$ value $<10 \times 10^{-10}$ ) between the three protein data sets (Ensembl Genomes, Release 22, for D. melanogaster and A. gambiae). Positions of pairwise orthologous genes were retrieved from the reference genomes (BDGP5, AgamP3 and CLUMA_1.0) and plotted with $\operatorname{Circos}^{61}$. C. marinus chromosome arms were delimited based on centromeric and telomeric signatures in genetic diversity and linkage disequilibrium (Extended Data Fig. 3c and Supplementary Table 3; for data source see 'strain re-sequencing' below). Homologues for C. marinus chromosome arms were assigned based on enrichment with putative orthologous genes from specific chromosome arms in D. melanogaster and A. gambiae (Extended Data Figs 3, 4 and Supplementary Table 3). Additionally, for the 5,388 detected putative 1:1:1 orthologues (C. marinus:D. melanogaster:A. gambiae), microsynteny was assessed by testing if all pairs of directly adjacent genes in one species were also directly adjacent in the other species. The degree of microsynteny was then calculated as the fraction of conserved adjacencies among all pairs of adjacent genes. From this fraction the relative levels of chromosomal rearrangements in the evolutionary lineage leading to C. marinus were estimated (Supplementary Note 3 and Extended Data Fig. 4).

Strain re-sequencing. Genetic variation in five C. marinus strains (Extended Data Fig. 1) was assessed based on pooled-sequencing data from field-caught males from the strains of St. Jean-de-Luz (Jean; Basque Coast, France; sampled in 2007; $n=300$ ), Port-en-Bessin (Por; Normandie, France; 2007; $n=300$ ), as well as Vigo (Spain; 2005; $n=100$ ), Helgoland (He; Germany; 2005; $n=300$ ) and Bergen (Ber; Norway; 2005; $n=100$ ). Samples from Vigo and Bergen, were provided by D. Neumann and C. Augustin, respectively. For each strain we chose the largest available number of individuals to obtain the best possible resolution of allele frequencies. Females are not available, because they are virtually invisible in the field. For an overview of the experimental procedure, see Extended Data Fig. 9b. DNA was extracted with a salting-out method ${ }^{46}$ from sub-pools of 50 males, the DNA pools were mixed at equal DNA amounts, sheared and prepared as described above and sequenced on four lanes of an Illumina HiSeq2000 with paired-end 100-bp reads (Ber and Vigo combined in one lane, distinguished by index reads). All reads were submitted to the European Nucleotide Archive (ENA) under project PRJEB8339. Sequencing reads were filtered for read quality and adaptor sequences with cutadapt ${ }^{47}(-\mathrm{b}-\mathrm{n} 2-\mathrm{e} 0.1-\mathrm{O} 8-\mathrm{q} 13-\mathrm{m} 15)$, interleaved with ngm-utils ${ }^{49}$ and duplicates were removed with fastq-mcf from ea-utils ${ }^{48}(-\mathrm{D} 70)$. Reads were aligned to the mapped super-scaffolds of assembly CLUMA_1.0 with BWA ${ }^{50}$ (aln and sampe; maximal insert size (bp): - a 1500).

Detection of re-arrangements. Based on the unfiltered alignments, the samples from Por and Jean were screened for genomic inversions and indels relative to the reference sequence with the multi-sample version of DELLY ${ }^{62}$. Paired-end information was only considered if the mapping quality was high $(q \geq 20)$ (see also Supplementary Note 3)

Population genomic analysis of the timing strains. For population genomic analysis (Extended Data Fig. 9b), the alignments of the pool-sequencing (pool-seq) data from Vigo, Jean, Por, He and Ber were filtered for mapping quality $(q \geq 20)$, sorted, merged and indexed with SAMtools ${ }^{63}$. Reads were re-aligned around indels with the RealignerTargetCreator and the IndelRealigner in GATK ${ }^{64}$. The resulting coverage per strain is given in Supplementary Table 5.

For identification of SNPs, a pileup file was created with the mpileup command of SAMtools ${ }^{63}$. Base Alignment Quality computation was disabled (-B); instead, after creating a synchronized file with the mpileup2sync script in PoPoolation $2^{65}$, indels that occurred more than ten times were masked (including 3 bp upstream and downstream) with the identify-indel-regions and filter-sync-by-gtf scripts of PoPoolations2. $F_{\mathrm{ST}}$ values were determined with the fst-sliding script of PoPoolation2, applying a minimum allele count of 10 (so that any false-positive SNPs resulting from the remaining unmasked indels were effectively excluded) and a minimum coverage of $40 \times$ for the comparison between Por and Jean or $10 \times$ for the comparison of all five strains. $F_{\mathrm{ST}}$ was calculated at a single base resolution, as well as in windows of $5 \mathrm{~kb}$ (step size, $1 \mathrm{~kb}$ ). Individual SNPs were only considered for further analyses or plotted if they were significantly differentiated as assessed by Fisher's exact test (fisher-test in PoPoolation2).

Average genome-wide genetic differentiation between timing strains, as obtained by averaging over 5 -kb sliding-windows, was compared to the respective timing differences and geographic distances (see Supplementary Table 8) in Mantel tests (Pearson's product moment correlation; 9,999 permutations), as implemented in the vegan package in the $\mathrm{R}$ statistical programming environment (ref. 66). Geographic distances and circadian timing differences were determined as described previously ${ }^{67}$ (see Supplementary Table 8). For determination of lunar timing differences when comparing lunar with semilunar rhythms see Supplementary Note 6. In order to find genomic regions for which genetic differentiation is correlated with the timing differences between strains, the Mantel test was then applied to $5-\mathrm{kb}$ genomic windows every $1 \mathrm{~kb}$ along the reference sequence. $5 \mathrm{~kb}$ is roughly the average size of a gene locus in C. marinus. Windows with a correlation coefficient of $r \geq 0.5$ were tested for significance ( 999 permutations) For each genomic position the number of overlapping significantly correlated 5-kb windows was enumerated, resulting in a correlation score (CS; ranging from 0 to 5 ).

Genetic diversity, measured as Watterson's theta $\left(\theta_{\mathrm{W}}\right)$, for each strain was assessed with PoPoolation1.1.2 (ref. 68) in 20-kb windows with 10-kb steps. In order to save computing time, the pileup files of Jean, Por and He were linearly downscaled to $100 \times$ coverage with the subsample-pileup script ('fraction' option), positions below $100 \times$ coverage were discarded. Indel regions were excluded (default in PoPoolation 1.1.2) and a minimum of $66 \%$ of a sliding window needed to be covered. SNPs were only considered in $\theta_{\mathrm{W}}$ calculations if present $\geq 2$ times, leading to slight inconsistencies in $\theta_{\mathrm{W}}$ estimates between strains due to differing coverage, but not affecting diversity comparisons within strains.

Linkage disequilibrium between the SNPs was determined for the Por and Jean strains with $\mathrm{LDx}^{69}$, assuming physical linkage between alleles on the same read or read pairs. $r^{2}$ was determined by a maximum likelihood estimator, minimum and maximum read depths corresponded to the $2.5 \%$ and $97.5 \%$ coverage depths for each population (Jean, 111-315; Por, 98-319), total insert distance was limited to $600 \mathrm{bp}$, minimum phred-scaled base quality was 20 , minimum allele frequency was 0.1 and a minimum coverage per pair of SNPs was 11 . SNPs were binned by their physical distance for the plots (0-200 bp, 200-400 bp, 400-600 bp), with the mean value plotted.

Finally, small indels $(<30 \mathrm{bp})$ in the Por and Jean strains were detected with the UnifiedGenotyper ( - glm INDEL) in GATK $^{64}$ for positions with more than $20 \times$ coverage. Genetic differentiation for indels was calculated with the classical formula $F_{\mathrm{ST}}=\left(H_{\mathrm{T}}-H_{\mathrm{S}}\right) / H_{\mathrm{T}}$, where $H_{\mathrm{S}}$ is the average expected heterozygosity according to Hardy-Weinberg Equilibrium (HWE) in the two subpopulations and $H_{\mathrm{T}}$ is the expected heterozygosity in HWE of the hypothetical combined total population. If more than two alleles were present, only the two most abundant alleles were considered in the calculation of $F_{\mathrm{ST}}$

Assessment of candidate genes. Gene models from the automated annotation were considered candidate genes, if they fulfilled the following criteria. (1) The gene was located within the reference sequence corresponding to the QTL confidence intervals as determined for the Por and Jean strains. (2) The gene contained a strongly differentiated SNP or small indel or it was directly adjacent to such a SNP or small indel ( $F_{\mathrm{ST}} \geq 0.8$ for Por versus Jean, that is, the strains used in QTL mapping). This resulted in a preliminary list of 133 genes based on the comparison between Por and Jean (Supplementary Table 6). These candidate genes were narrowed down based on their overlap with genomic 5-kb windows, for which genetic differentiation between five European timing strain correlated with their timing differences (Fig. 1a, Extended Data Fig. 5a, b and Supplementary Table 9).

The location and putative effects of the SNPs and indels relative to the gene models were assessed with SNPeff ${ }^{70}$ ( - ud 0 , otherwise default parameters; Extended Data Fig. 5c, d and Supplementary Tables 6, 9).

For Gene Ontology (GO) term analysis, all C. marinus gene models with putative orthologues in the UniProtKB/Swiss-Prot and non-redundant protein sequences (nr) databases based on reciprocal best BLAST hits (see above) were annotated with the GO terms of their detected orthologues (6,837 gene models). Paralogues were not annotated. The enrichment of candidate SNPs and indels $\left(F_{\mathrm{ST}} \geq 0.8\right.$ between Por and Jean) in specific GO terms was tested with SNP2GO ${ }^{71}$ (min.regions $=1$, otherwise default parameters). Hyper-geometric sampling was applied to test if individual genes of a GO term or a whole pathway of genes are enriched for SNPs (Supplementary Table 7).

Molecular characterization of CaMKII.1. RNA-seq data of the Por and Jean strains for CaMKII. 1 were obtained from the larval RNA sequencing experiment described above. Besides four assembled full-length transcripts (RA-RD) from RNA-seq and assembled EST libraries, additional partial transcripts (RE-RO) were identified by PCR amplification (for PCR primers see Supplementary Table 15), gel extraction (QIAquick Gel Extraction Kit, Qiagen), cloning with the CloneJET PCR Cloning Kit (Thermo Scientific) and Sanger sequencing with pJET1.2 primers (LGC Genomics \& Microsynth). cDNA was prepared from RNA extracted from third instar larvae of the Por and Jean laboratory strains (RNA extraction with RNeasy Plus Mini Kit, Qiagen; reverse transcription with QuantiTect Reverse Transcription Kit, Qiagen)

qPCR was performed with variant-specific primers and actin was used as a control gene (Supplementary Table 16). cDNA was obtained from independent pools of 20 third instar larvae of the Por and Jean strains. Sample size was ten pools per strain to cover different time points during the day and to test for reproducibility 
(two samples each at zeitgeber times $0,4,8,16$ and 20; for one Por sample extraction failed; RNA extraction and reverse transcription as above). qPCR was performed with Power SYBR Green PCR Master Mix on a StepOnePlus Real Time System (both Applied Biosystems). Fold-changes were calculated according to ref. 72 in a custom excel sheet. The assumption of equal variance was violated for the $\mathrm{RD}$ comparison ( $F$-test) and the assumption of normal distribution was violated for the data of RA and RC in the Por strain (Shapiro-Wilk normality test), possibly reflecting circadian effects in the samples from different times of day. Thus, expression differences were assessed for significance in a two-tailed Wilcoxon rank-sum test (wilcox.test in $\mathrm{R}^{66}$ ). Holm correction ${ }^{73}$ was used for multiple testing (default in p.adjust function of $\mathrm{R}$ ).

CaMKII.1 minigenes. PCR fragments containing the CaMKII.1 linker region (exons 10-15) were amplified from genomic Por or Jean DNA, respectively, with primers CaMKII-Sc61-F-344112 and CaMKII-Sc61-R-351298 (Supplementary Table 15), cloned with the CloneJET PCR Cloning Kit (Thermo Scientific), transferred into the pcDNA3.1+ vector using NotI and XbaI (Thermo Scientific). These constructs were transfected into D. melanogaster $\mathrm{S} 2 \mathrm{R}+$ cells and RNA was prepared $48 \mathrm{~h}$ after transfection. After DNase digestion, isoform expression was analysed by radioactive, splicing-sensitive RT-PCR (primers in Supplementary Table 17) and phosphorimager quantification as described ${ }^{74}$. Identity of isoforms is based on size and sequencing of PCR products. To test for reproducibility, there were seven biological replicates (raw data in Supplementary Table 18). As the assumptions of equal variance $(F$-test) and normal distribution of data (Shapiro-Wilk normality test) were not violated, the significance of expression differences was assessed in unpaired, two-sided two-sample $t$-tests. Holm correction ${ }^{73}$ was used for multiple testing (default in p.adjust function of R). S2R+ cells were obtained from the laboratory of S. Sigrist, regularly authenticated by morphology and routinely tested for absence of mycoplasma contamination. The entire experiment was reproduced several months later with three biological replicates (raw data in Supplementary Table 18).

S2 cell luciferase assay. Firefly luciferase is driven from a period 3X69 promoter under control of the CLOCK and CYCLE protein ${ }^{19,21}$. The D. melanogaster pAc-clk construct was obtained from $\mathrm{F}$. Rouyer, $p$ Copia-Renilla luciferase and period 3X69-luc reporter constructs from M. Rosbash, a $\left[\mathrm{Ca}^{2+}\right]$-independent mouse CaMKII ${ }^{\mathrm{T} 286 \mathrm{D}}$ was provided by M. Mayford. The CaMKII inhibitor KN-93 was purchased from Abcam (\#ab120980).

C. marinus Cyc, C. marinus Clk and C. marinus CaMKII.1-RD were cloned into the pAc5.1/V5-His A plasmid (Invitrogen) with stop codons before the tag. The Q5 Site-Directed Mutagenesis Kit (NEB) was used to make kinase-dead and $\left[\mathrm{Ca}^{2+}\right]$-independent versions of C. marinus CaMKII.1-RD (for primers, see Supplementary Table 17).

D. melanogaster S2 cells (Invitrogen) were cultured at $25^{\circ} \mathrm{C}$ in Schneider's D. melanogaster medium (Lonza) supplemented with fetal bovine serum (FBS, $10 \%$, heat-inactivated), penicillin $\left(100 \mathrm{U} \mathrm{ml}^{-1}\right)$, streptomycin $\left(100 \mu \mathrm{g} \mathrm{ml}^{-1}\right)$ and $2 \mathrm{mM}$ L-glutamine; Sigma). Cells were seeded into 24 -well plates $(800,000$ cells per well) and transfected with Effectene transfection reagent (Qiagen) according to the manufacturer's instructions. Experiment with mouse $\left[\mathrm{Ca}^{2+}\right]$-independent CaMKII: $25 \mathrm{ng}$ pCopia-Renilla, $10 \mathrm{ng}$ period 3 X69-luc, $0.5 \mathrm{ng}$ D. melanogaster $p A c-c l k, 200$ ng mouse $p A c-C a M K I I^{\mathrm{T} 286 \mathrm{D}}$. Experiment with CaMKII inhibitor KN-93: 25 ng pCopia-Renilla, 10 ng period 3X69-luc, 0.5 ng D. melanogaster $p A c-c l k$, various amounts of KN-93. Experiment with C. marinus genes: $25 \mathrm{ng}$ pCopia-Renilla, $10 \mathrm{ng}$ period 3X69-luc, $100 \mathrm{ng}$ C. marinus $p A c-c y c, 100 \mathrm{ng}$ C. marinus $p A c-c l k, 200 \mathrm{ng}$ C. marinus CaMKII.1-RD ${ }^{\mathrm{K} 42 \mathrm{R}}$ or $200 \mathrm{ng}$ C. marinus $C a M K I I .1-R D^{\mathrm{T} 286 \mathrm{D}}$. In all experiments, the transfection mix was filled up with empty pAc5.1/V5-His A vector to a total of $435 \mathrm{ng}$ DNA per well. After $48 \mathrm{~h}$, cells were washed with PBS and lysed with Passive Lysis Buffer (Promega). Luciferase activities were determined on a Synergy H1 plate reader (Biotek) using a DualLuciferase Reporter Assay System (Promega). For each biological replicate three independent cell lysates were measured and their mean value determined. Firefly luciferase activity was normalized to Renilla luciferase activity and values were normalized to controls transfected with D. melanogaster $p A c-c l k$ or $C$. marinus $p A c-c l k$ and C. marinus $p A c-c y c$, respectively. S2 cells (Invitrogen/Life Technologies, Cat.no. R690-07) were regularly authenticated by morphology and routinely tested for absence of mycoplasma contamination (Lonza MycoAlert). Sample size was chosen to test for reproducibility.

Circadian free-run experiments. For circadian free-run experiments, culture boxes of the Por, He and Jean strains were transferred from light-dark cycle (16:8) to constant dim light (light-light cycle, about $100 \mathrm{~lx}$ ). Emerging adults were collected in 1-h intervals by a custom made C. marinus fraction collector (similar to those described in ref. 75) and counted once a day. Because collection was automated, the experimenter had no influence on the results and blinding was not necessary. As the circalunar clock restricts adult emergence to a few days, the circadian emergence rhythm can only be assessed over a few days. Several culture boxes were transferred to a light-light cycle at different time points. The resulting emergence data were combined for each strain using the switch to a light-light cycle as a common reference point. We used the maximum number of available individuals. Free-running period was calculated as the mean interval between subsequent emergence peaks, weighting each peak by the number of individuals. Data availability. All sequence data are deposited in the European Nucleotide Archive (ENA) under PRJEB8339. The reference genome is also on ClunioBase (http://cluni obase.cibiv.univie.ac.at). Machine readable super-scaffolding data and the computer source code for the removal of repeated edges are supplied as source data files.

46. Reineke, A., Karlovsky, P. \& Zebitz, C. P. W. Preparation and purification of DNA from insects for AFLP analysis. Insect Mol. Biol. 7, 95-99 (1998).

47. Martin, M. Cutadapt removes adapter sequences from high-throughput sequencing reads. EMBnet.journal 17, 10-12 (2011).

48. Aronesty, E. Command-line tools for processing biological sequencing data http://code.google.com/p/ea-utils (2011).

49. Sedlazeck, F. J., Rescheneder, P. \& von Haeseler, A. NextGenMap: fast and accurate read mapping in highly polymorphic genomes. Bioinformatics $\mathbf{2 9}$ 2790-2791 (2013)

50. Li, H. \& Durbin, R. Fast and accurate short read alignment with BurrowsWheeler transform. Bioinformatics 25, 1754-1760 (2009).

51. Zerbino, D. R. \& Birney, E. Velvet: algorithms for de novo short read assembly using de Bruijn graphs. Genome Res. 18, 821-829 (2008)

52. Gladman, S. \& Seemann, T. VelvetOptimiser http://bioinformatics.net.au/ software.velvetoptimiser.shtml (2012)

53. Boetzer, M., Henkel, C. V., Jansen, H. J., Butler, D. \& Pirovano, W. Scaffolding pre-assembled contigs using SSPACE. Bioinformatics 27, 578-579 (2011).

54. Barton, M. D. \& Barton, H. A. Scaffolder-software for manual genome scaffolding. Source Code Biol. Med. 7, 4 (2012).

55. Boetzer, M. \& Pirovano, W. Toward almost closed genomes with GapFiller. Genome Biol. 13, R56 (2012)

56. Andrews, S. FastQC Version 0.10.1. A Quality Control tool for High Throughput Sequence Data http://www.bioinformatics.babraham.ac.uk/projects/fastqc/ (2012).

57. Grabherr, M. G. et al. Full-length transcriptome assembly from RNA-Seq data without a reference genome. Nat. Biotechnol. 29, 644-652 (2011).

58. Holt, C. \& Yandell, M. MAKER2: an annotation pipeline and genome-database management tool for second-generation genome projects. BMC Bioinformatics 12, 491 (2011)

59. Stanke, M. \& Waack, S. Gene prediction with a hidden Markov model and a new intron submodel. Bioinformatics 19 (Suppl 2), ii215-ii225 (2003).

60. Korf, I. Gene finding in novel genomes. BMC Bioinformatics 5, 59 (2004).

61. Krzywinski, M. et al. Circos: an information aesthetic for comparative genomics. Genome Res. 19, 1639-1645 (2009)

62. Rausch, T. et al. DELLY: structural variant discovery by integrated paired-end and split-read analysis. Bioinformatics 28, i333-i339 (2012).

63. Li, H. et al. The sequence alignment/map format and SAMtools. Bioinformatics 25, 2078-2079 (2009)

64. McKenna, A. et al. The genome analysis toolkit: a MapReduce framework for analyzing next-generation DNA sequencing data. Genome Res. 20, 1297-1303 (2010)

65. Kofler, R., Pandey, R. V. \& Schlötterer, C. PoPoolation2: identifying differentiation between populations using sequencing of pooled DNA samples (Pool-Seq). Bioinformatics 27, 3435-3436 (2011).

66. Crawley, M. J. The R Book. (John Wiley \& Sons Ltd., 2007).

67. Kaiser, T. S., Neumann, D., Heckel, D. G. \& Berendonk, T. U. Strong genetic differentiation and postglacial origin of populations in the marine midge Clunio marinus (Chironomidae, Diptera). Mol. Ecol. 19, 2845-2857 (2010).

68. Kofler, R. et al. PoPoolation: a toolbox for population genetic analysis of next generation sequencing data from pooled individuals. PLoS One 6, e15925 (2011).

69. Feder, A. F., Petrov, D. A. \& Bergland, A. O. LDx: estimation of linkage disequilibrium from high-throughput pooled resequencing data. PLoS One 7, e48588 (2012)

70. Cingolani, P. et al. A program for annotating and predicting the effects of single nucleotide polymorphisms, SnpEff: SNPs in the genome of Drosophila melanogaster strain w1118; iso-2; iso-3. Fly (Austin) 6, 80-92 (2012)

71. Szkiba, D., Kapun, M., von Haeseler, A. \& Gallach, M. SNP2GO: functional analysis of genome-wide association studies. Genetics 197, 285-289 (2014).

72. Livak, K. J. \& Schmittgen, T. D. Analysis of relative gene expression data using real-time quantitative PCR and the $2^{(-\Delta \Delta C(T)}$ method. Methods 25, 402-408 (2001)

73. Holm, S. A simple sequentially rejective multiple test procedure. Scand. J. Stat. 6, 65-70 (1979)

74. Preußner, M. et al. Rhythmic U2af26 alternative splicing controls PERIOD1 stability and the circadian clock in mice. Mol. Cell 54, 651-662 (2014).

75. Honegger, H. W. An automatic device for the investigation of the rhythmic emergence pattern of Clunio marinus. Int. J. Chronobiol. 4, 217-221 (1977).

76. Heimbach, F. Semilunare und diurnale Schlüpfrhythmen südenglischer und norwegischer Clunio-Populationen (Diptera, Chironomidae) PhD thesis, Universität Köln (1976).

77. Friedrich, M. \& Tautz, D. Evolution and phylogeny of the Diptera: a molecular phylogenetic analysis using 28S rDNA sequences. Syst. Biol. 46, 674-698 (1997)

78. Zdobnov, E. M., et al. Comparative genome and proteome analysis of Anopheles gambiae and Drosophila melanogaster. Science 298, 149-159 (2002). 
a

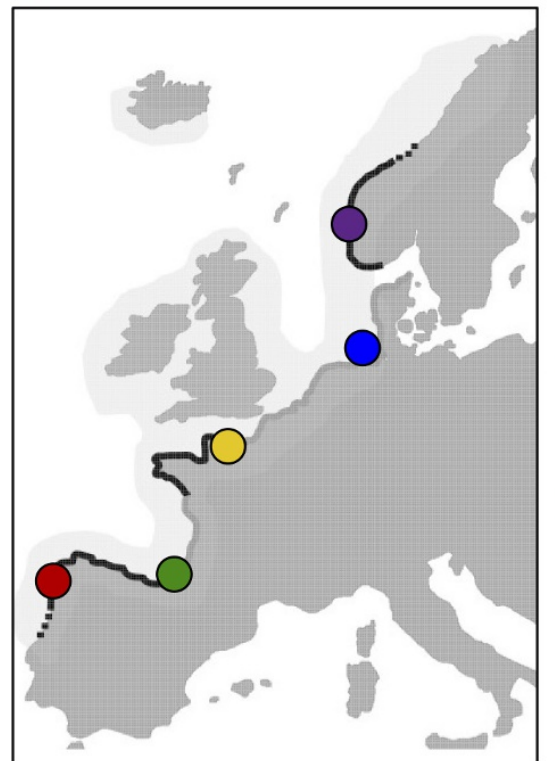

Extended Data Figure 1 | The biology of Clunio marinus. a, C. marinus is restricted to rocky shores (black lines), the localities differing in tidal regime (adapted from ref. 67). b, c, Local strains show corresponding genetic adaptations in their circadian ${ }^{67}(\mathbf{b})$ and circalunar rhythms $\left(\mathbf{c}, \mathrm{He}^{1}\right.$, $J e^{5}{ }^{5}$. Timing was measured in the laboratory under artificial moonlight

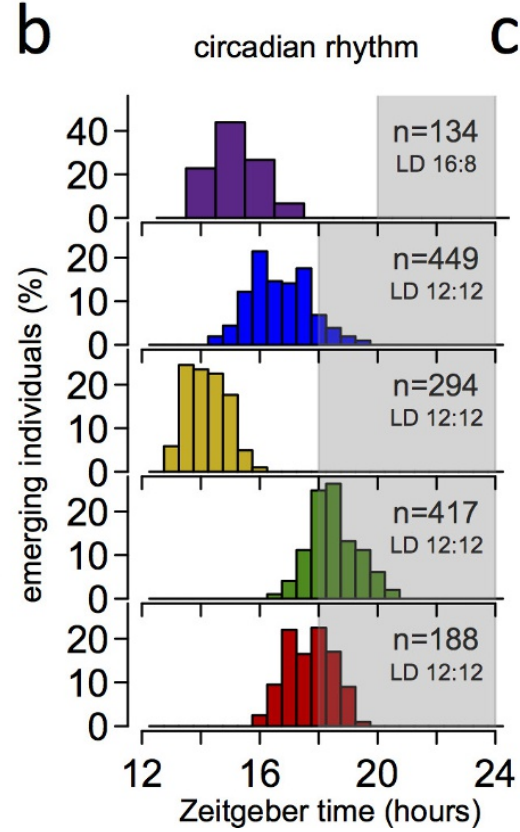

C circalunar rhythm

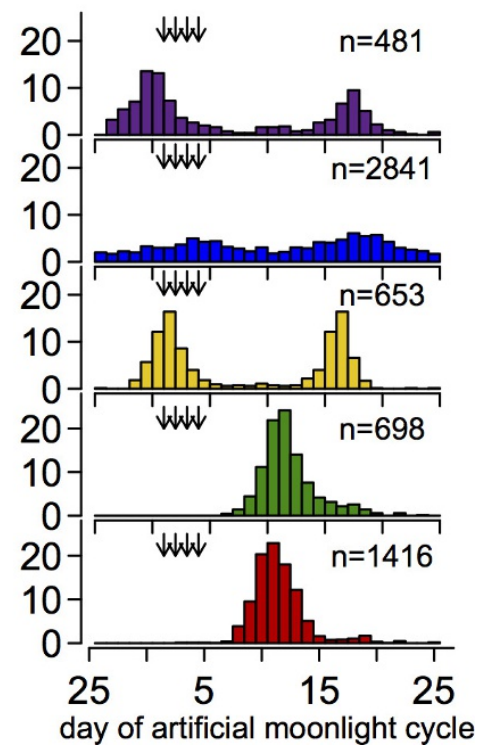

(arrows in c) in a 30-day cycle and a light-dark cycle of 12:12 (He, Por, Jean, Vigo) or 16:8 (Ber). Seasonal differences in daily illumination duration do not affect circadian emergence peaks ${ }^{1,76}$. Historically, for C. marinus 'zeitgeber time 0 ' is defined as the middle of the dark phase. 


\section{RESEARCH ARTICLE}
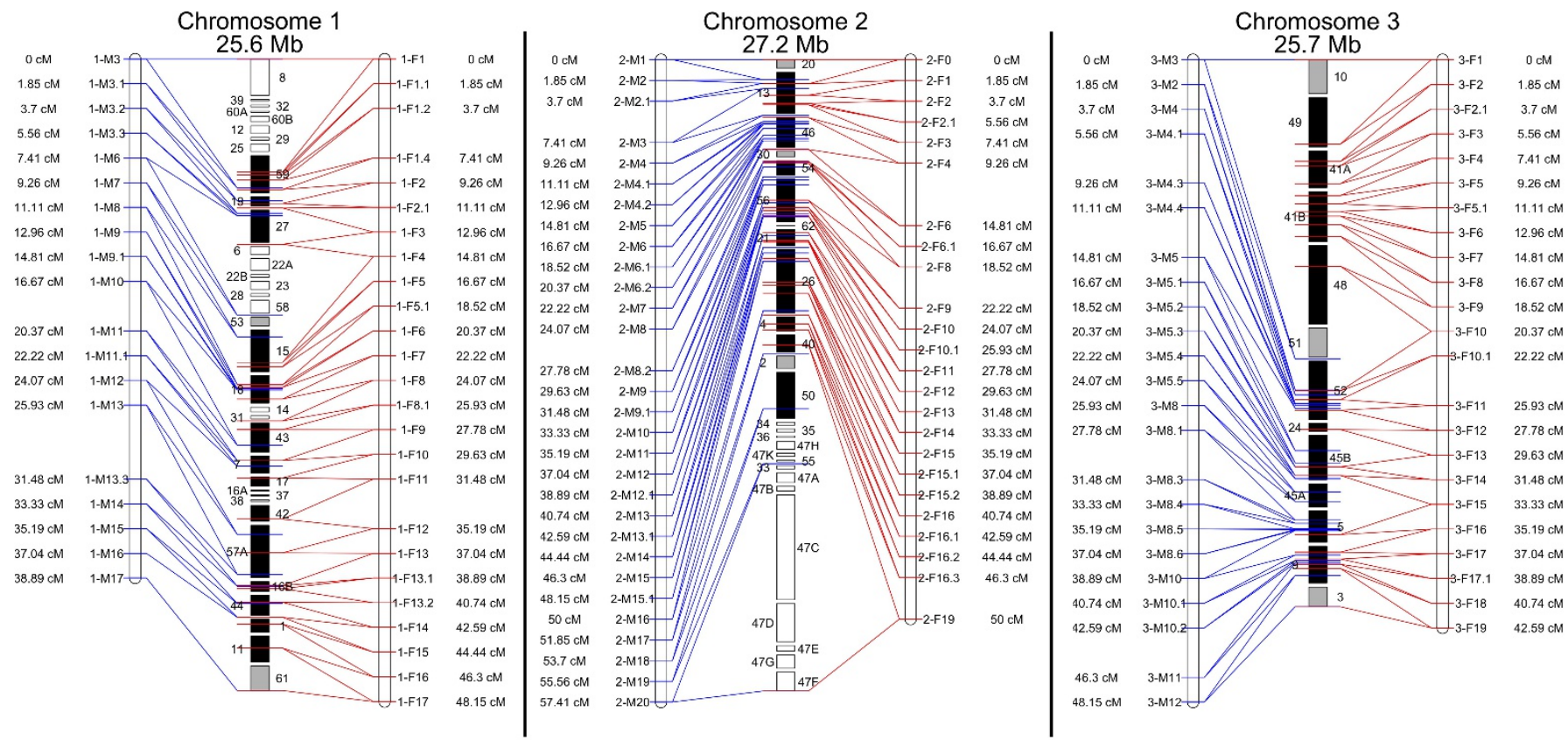

$\square$ mapped, ordered, oriented (53.6 Mb; $62.65 \%$ )
mapped, ordered, not oriented ( $7.3 \mathrm{Mb} ; 8.57 \%)$
mapped, unordered and not oriented ( $17.6 \mathrm{Mb} ; 20.58 \%$ )

$78.5 \mathrm{Mb} ; 91.80 \%$

unmapped $(7.0 \mathrm{Mb} ; 8.20 \%)$

Extended Data Figure $2 \mid$ The reconstructed chromosomes of $C$. marinus based on the genetic linkage map. Left map, male informative markers. Right map, female informative markers. See Fig. 1a legend for further details. 
a

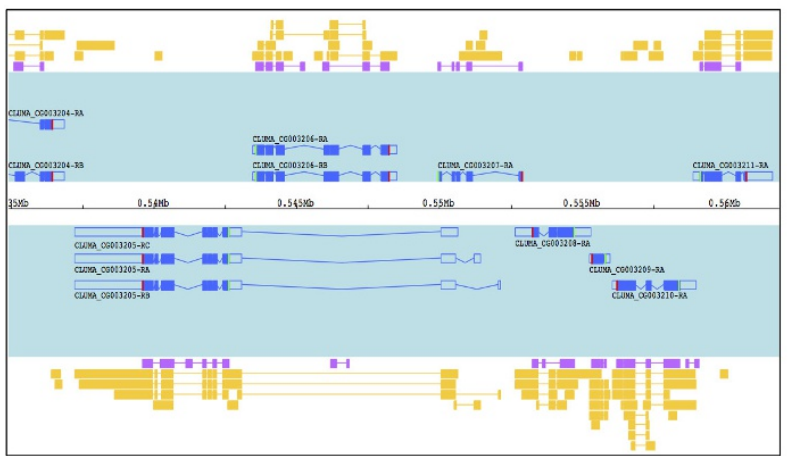

b

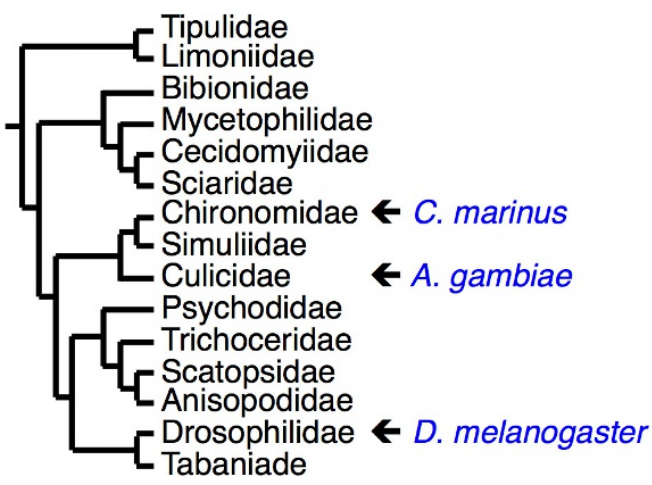

C
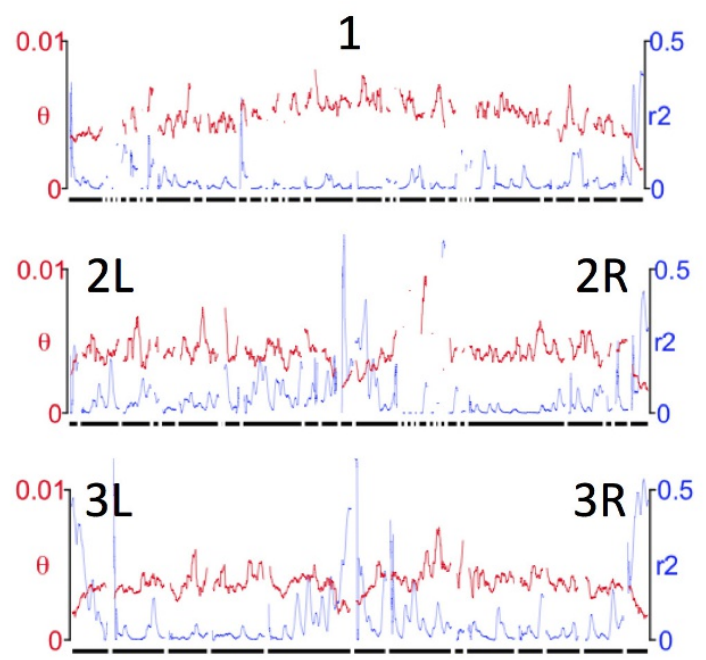

position along linkage group

Extended Data Figure $3 \mid$ C. marinus genome characterization. a, Representative genomic region with densely packed gene models (super-scaffold 1, 535-565 kb). Gene models are shown in blue on a turquoise background. Gene predictions (SNAP) are shown in purple. Transcript evidence is shown in yellow. $\mathbf{b}$, Phylogenetic relationships of C. marinus to other Diptera (according to ref. 77). c, Genetic diversity d

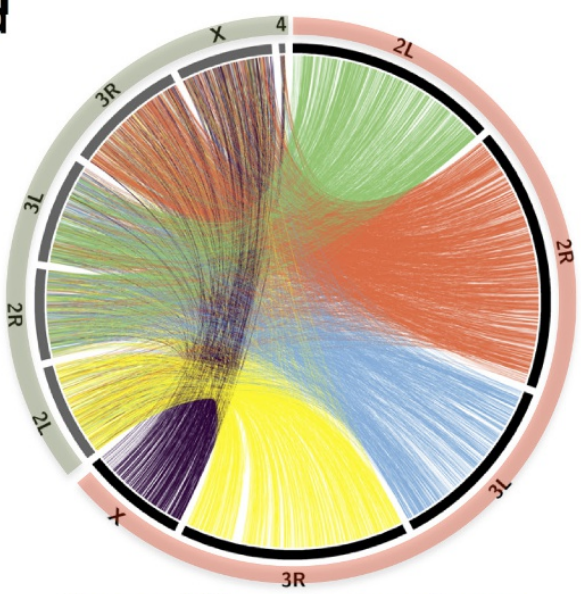

Drosophila melanogaster vs. Anopheles gambiae

e

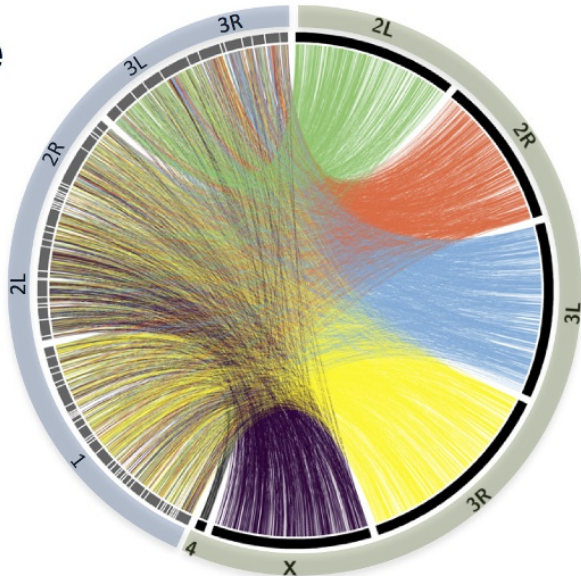

Clunio marinus vs. Drosophila melanogaster

f

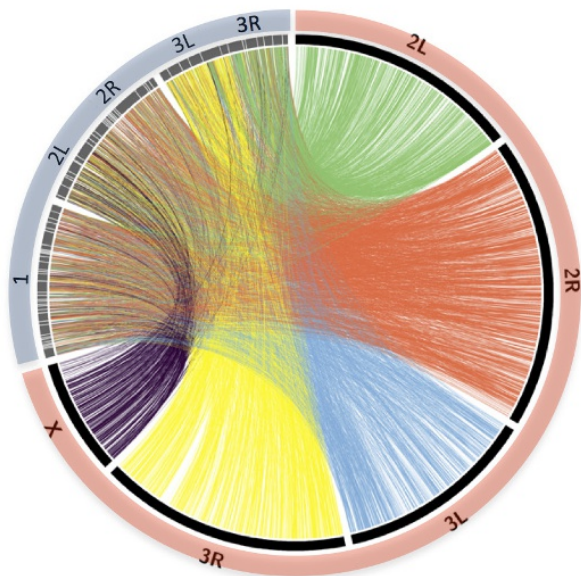

Clunio marinus vs. Anopheles gambiae

$(\theta$; red $)$ and linkage disequilibrium $\left(r^{2}\right.$; blue) of the Jean strain plotted for the three $C$. marinus linkage groups, revealing characteristic signatures of telomeres and centromeres. $\mathbf{d}-\mathbf{f}$, Synteny comparisons among the genomes of C. marinus, A. gambiae and D. melanogaster based on 5,388 1:1:1 orthologues. 
a
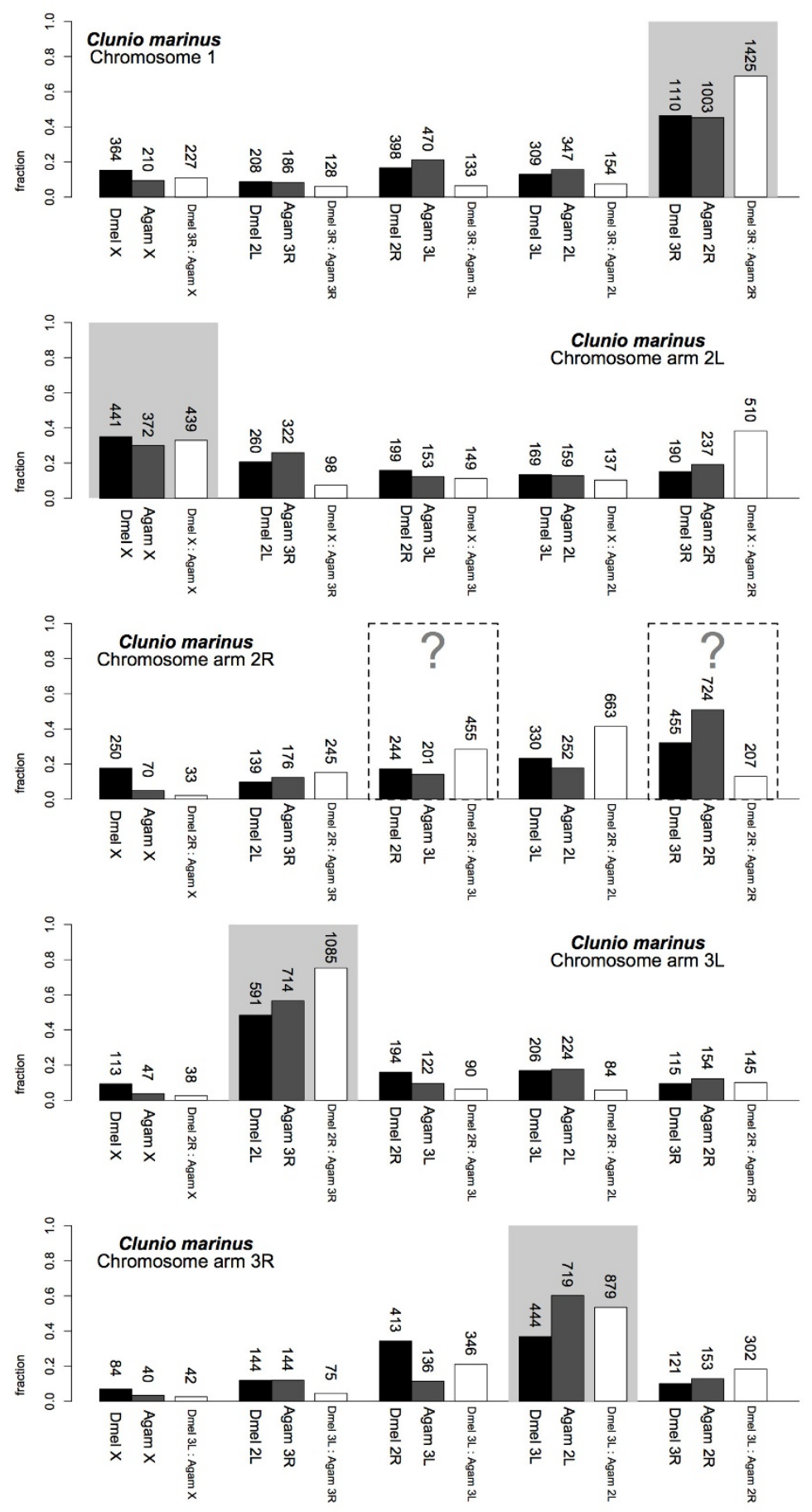

b

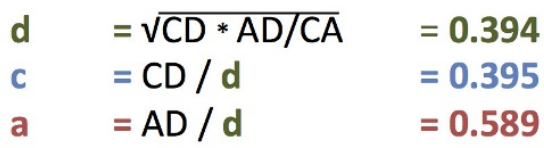

\section{Clunio} marinus

\section{Anopheles Drosophila gambiae melanogaster}

\section{C}

\section{Randomly placed breaks (rearrangements)}
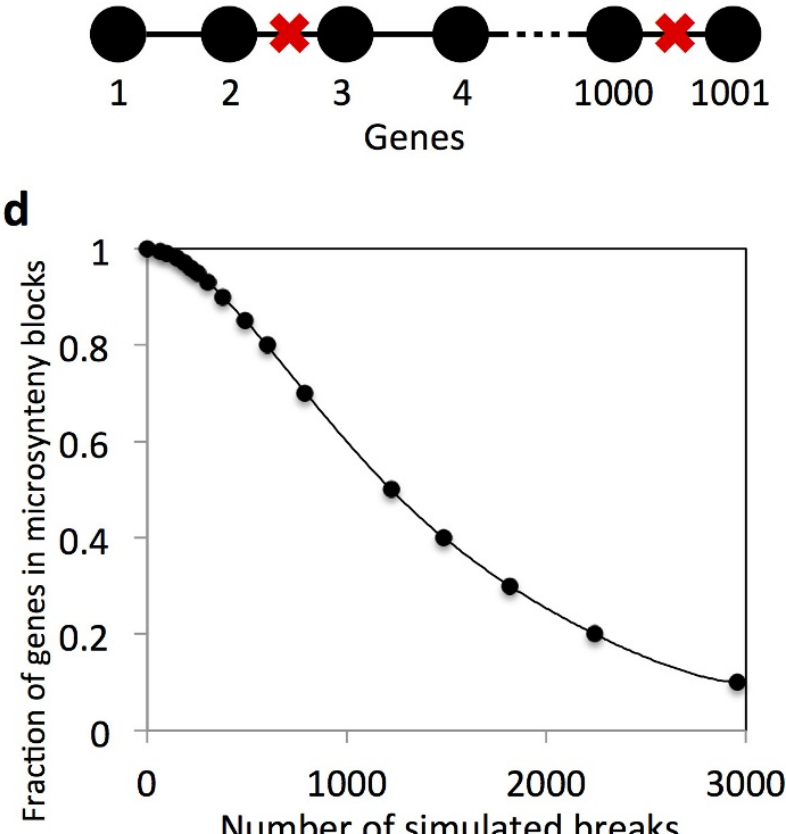

Extended Data Figure 4 | Synteny analyses of $C$. marinus chromosome arms. a, Gene content of the $C$. marinus chromosome arms relative to the chromosome arms of D. melanogaster (black bars) and A. gambiae (grey bars). The very small chromosome 4 of $D$. melanogaster is neglected. Chromosome arms of D. melanogaster and A. gambiae are paired according to their published homology ${ }^{78}$. For four of the chromosome arms of C. marinus the homologous arms in D. melanogaster and A. gambiae are identified (grey shading). For comparison, the conservation of the identified D. melanogaster and A. gambiae homologues to each other is given by plotting the gene content of the homologous $D$. melanogaster chromosome arm relative to the different chromosome arms of A. gambiae (white bars). The numbers of orthologous genes considered in each comparison are given above the bars. For chromosome arm $2 \mathrm{R}$ of C. marinus the homologies are unclear. Possibly, chromosome arm $2 \mathrm{R}$ of C. marinus has undergone so many re-arrangements with other chromosome arms that it is no longer recognizable, which is consistent with complex polymorphic re-arrangements in this chromosome arm of C. marinus (see Supplementary Note 3). b, Microsynteny is analysed relative to D. melanogaster and A. gambiae, based on 5,388 1:1:1 orthologues. The fraction of genes in conserved microsynteny blocks is calculated and distributed along the phylogenetic tree. c, d, A simulation was used to estimate how many chromosomal re-arrangements are required to produce the observed degree of microsyntenic conservation (for details see Supplementary Note 3). 
a

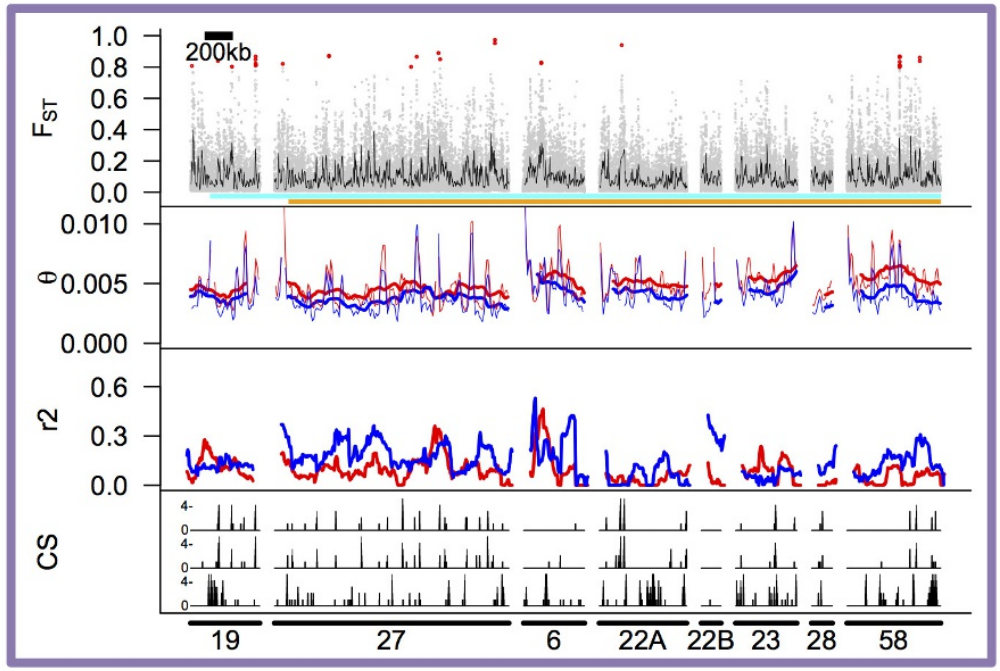

b

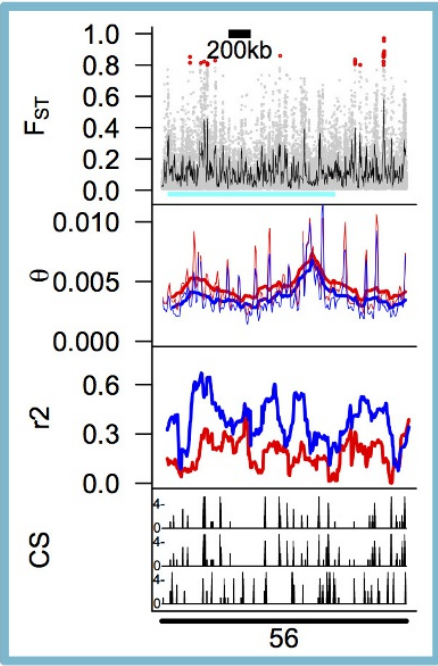

C

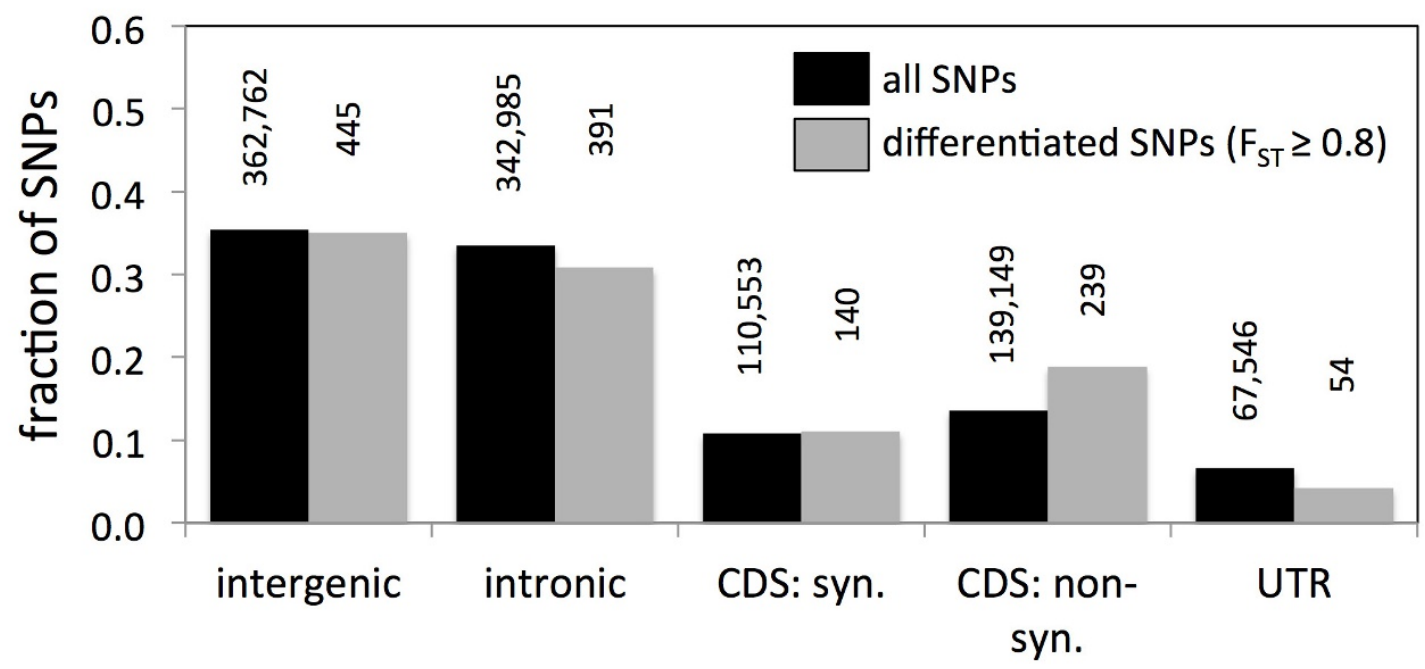

d

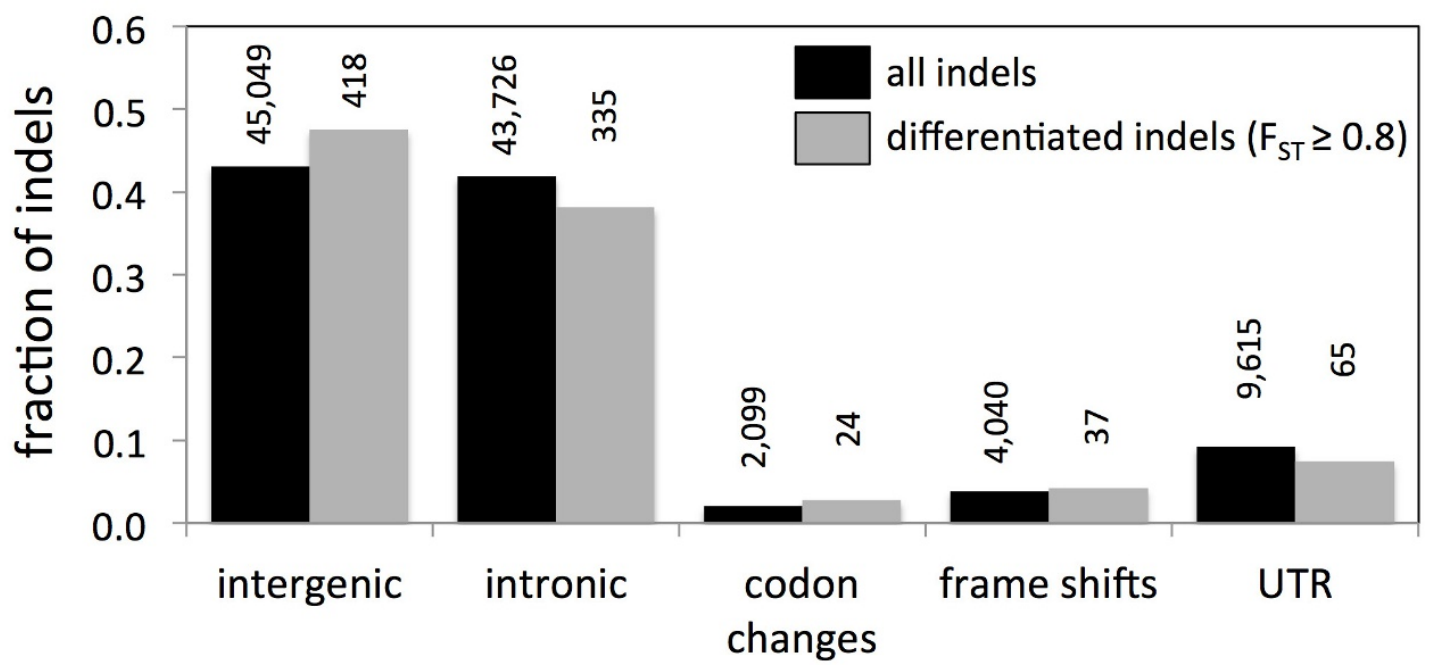

Extended Data Figure 5 | See next page for caption. 


\section{RESEARCH ARTICLE}

Extended Data Figure 5 | Population genomic analysis of QTLs C1/L1 and $\mathrm{C} 2$ and genome-wide analysis of locations and putative effects of SNPs and indels. a, b, Population genomic analysis of QTLs C1/L1 and C2. Panels 1-3: Por versus Jean strains in blue and red, respectively, in panel 2 and 3. From top to bottom, panel 1, genetic differentiation (red dots, SNPs with $F_{\mathrm{ST}} \geq 0.8$; grey dots, $F_{\mathrm{ST}}<0.8$; black line, average $F_{\mathrm{ST}}$ in 5 -kb sliding windows). Panel 2, genetic diversity $(\theta)$ in 20-kb (thin line) and $200-\mathrm{kb}$ (thick line) windows. Panel 3, linkage disequilibrium $\left(r^{2}\right)$ for SNP pairs 0-600 bp apart in 100-kb windows (step size: $5 \mathrm{~kb}$ ). Panel 4, correlation score (CS; $0-5)$ for genetic differentiation with circadian timing (top), circalunar timing (middle) and geographic distance (bottom) for five European C. marinus strains (Vigo, Jean, Por, He and Ber). Bottom numbers, scaffold IDs. See also Fig. 1. c, d, Locations and putative effects of SNPs (c) and indels (d) with respect to the annotated gene models. The fractions of SNPs or indels in each category are compared for all SNPs and indels (black bars) versus differentiated SNPs and indels $\left(F_{\mathrm{ST}} \geq 0.8\right.$ between Por and Jean strains; grey bars). Absolute numbers are given above the bars. In gene models with several splice forms, SNPs and indels can have different effects, for example, 'CDS, non-synonymous' for one splice form and 'intronic' for another splice form. Therefore, the sum across locations is slightly larger than the actual numbers of SNPs and indels. 'Codon changes' are all codon insertions or deletions that do not result in frame shifts beyond the actual insertion/deletion site. CDS, coding sequence; syn., synonymous; non-syn., non-synonymous; UTR, untranslated region. 


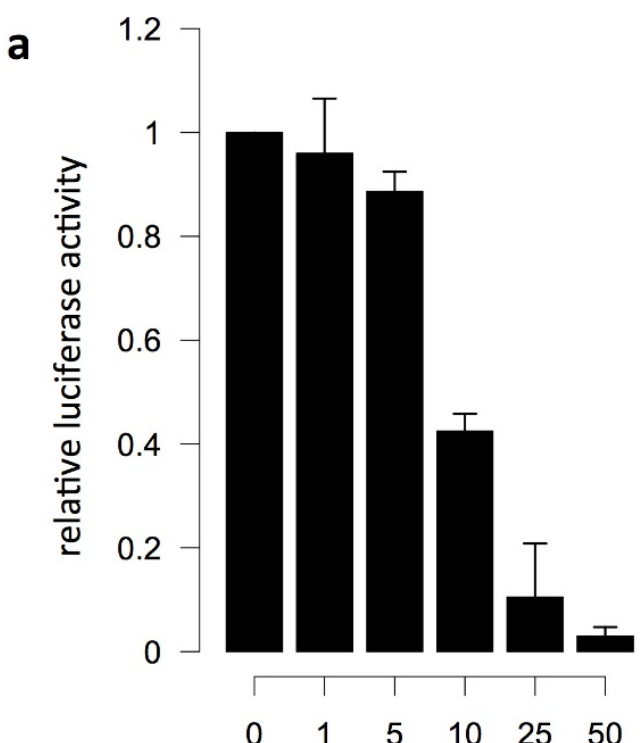

$\mathrm{KN}-93$ concentration $[\mu \mathrm{M}]$ b

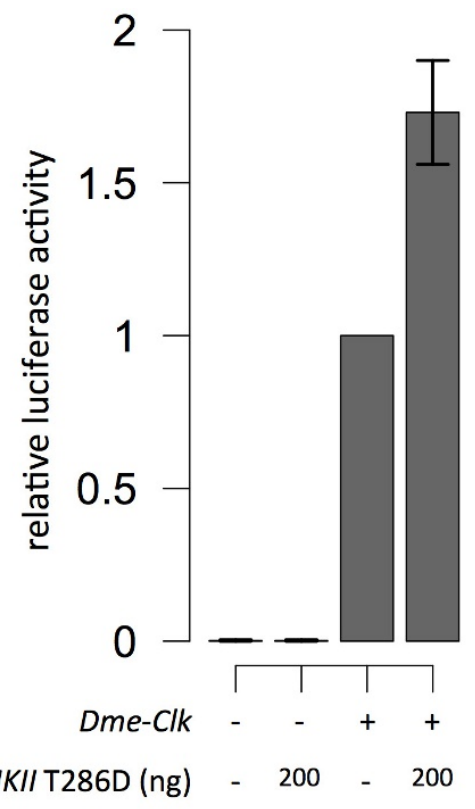

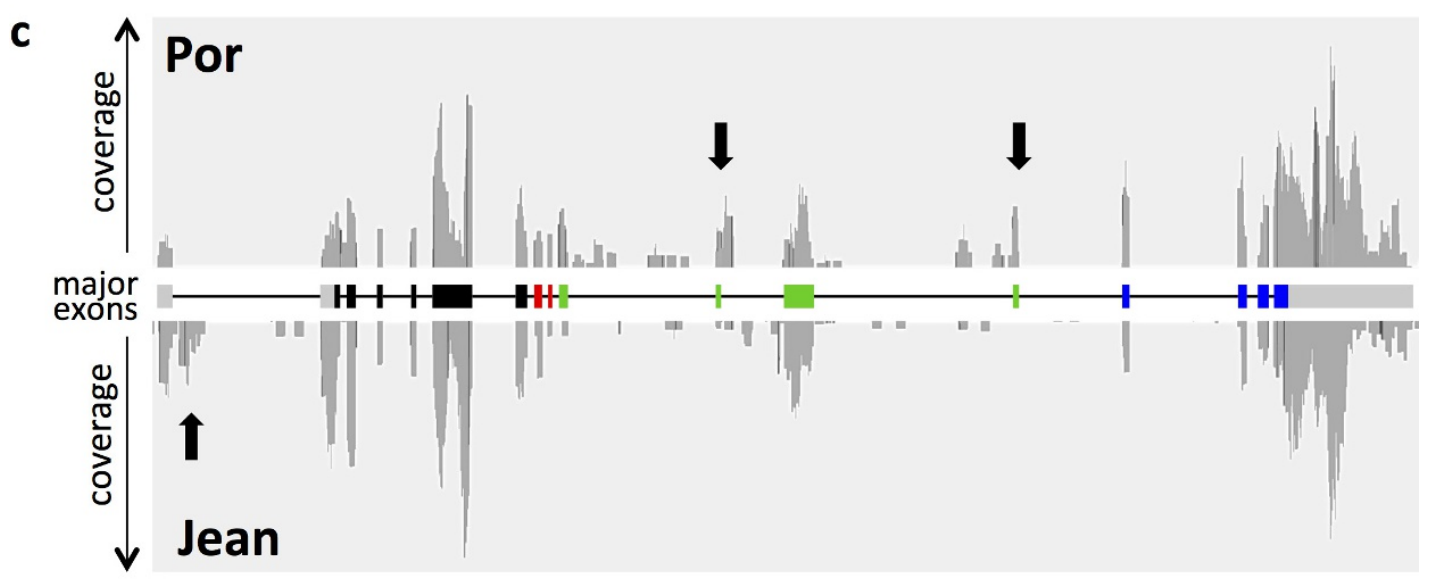

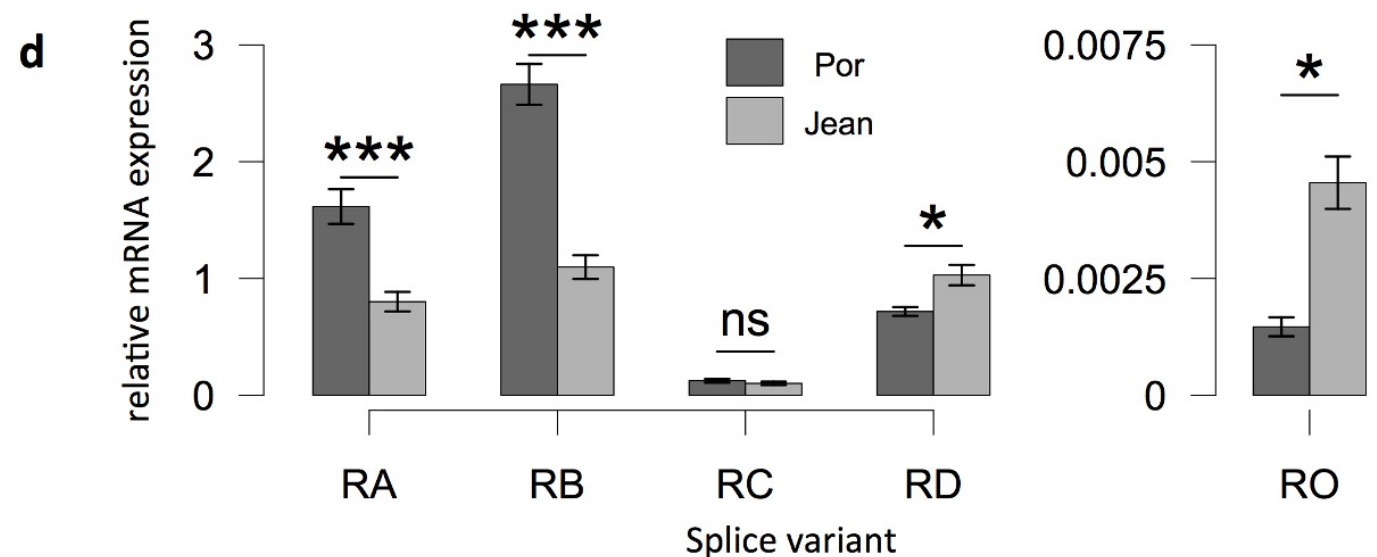

Extended Data Figure 6 | CaMKII regulates CLK/CYC transcriptional activity and exhibits strain-specific splice variants. a, Quantification of luciferase activity under the control of an artificial 3X69 E-box containing enhancer in D. melanogaster S2 cells. Increasing amounts of the CaMKII inhibitor KN-93 decrease luciferase activity in a concentration-dependent manner, providing evidence that endogenous CaMKII activity regulates the transcriptional activity of D. melanogaster CLOCK-CYCLE. b, Without co-transfection of D. melanogaster clock, there is no detectable luciferase activity. The constitutively active form of CaMKII (mouse T286D) increases luciferase activity (normalized to $\mathrm{CLOCK}^{+}$; data are shown as mean \pm s.e.m.; $n=4$ biological replicates). c, RNA sequencing reads mapped to the CaMKII.1 genomic locus. Arrows, major differences between the strains. d, Relative expression levels of the four major CaMKII.1 transcripts (RA-RD) and the minor variant RO in the Por and Jean strains of C. marinus, as measured by qPCR (data are shown as mean \pm s.e.m.; two-sided Wilcoxon rank-sum test; $* * * P<0.0005$; $* P<0.05$; NS, not significant; Holm correction for multiple testing; biological replicates, Por $n=9$, Jean $n=10$; except for RO: Por $n=3$, Jean $n=8)$. RO was not detectable in six additional biological replicates of the Por strain, suggesting that the expression differences are even greater than currently estimated. Figure 3 a shows the same data, normalized to the respective Por strain variants. 


\begin{tabular}{|c|c|c|c|c|c|}
\hline Por & $\begin{array}{l}\text { AGTGTGGAAG } \\
\text { AG TGTGGAAG }\end{array}$ & TAATTTTTAT & $\begin{array}{l}\text { СТСАСАТССА } \\
\text { СТСАСАGCCA }\end{array}$ & $\begin{array}{l}\text { TTAAGATTCT } \\
\text { TTAAGATTCT }\end{array}$ & $\begin{array}{l}\text { TATTGCTTTT } \\
\text { TATTGCTTTT }\end{array}$ \\
\hline $\begin{array}{l}\text { Po } \\
\text { Jear }\end{array}$ & TC & -TT & & ATGT & \\
\hline
\end{tabular}

Por tCTAgCATAg GTCTGCCATA aggctaAaAa aAATtgacag tTgCcGatag Jean

Por GTCATCCCCA CGGTGCCACA TGGTGCATTT GTGCCGCTCG GGCTTATACA Jean

Por TTGTACATTC ATTGCACATT ATAAATCCAT AATTTTTAAT TTGATATCTA Jean TTGTACATTC ATTGCACATT ATAAATCCAT AATTTTTAAT TTGATATCTA
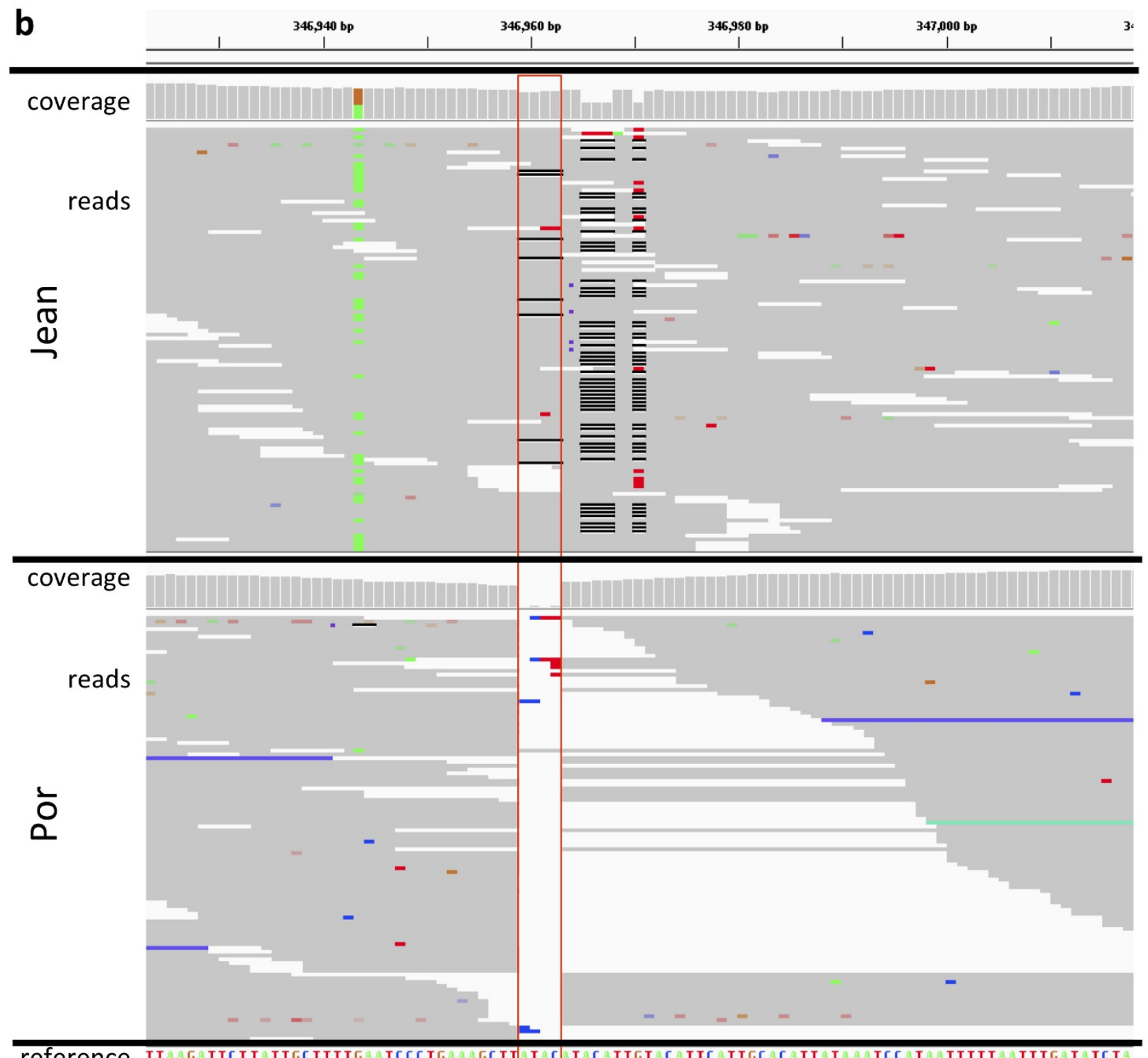

Extended Data Figure $7 \mid$ A differentiated 125-bp insertion in the CaMKII locus. a, Alignment of the part of the CaMKII locus of the Por and Jean strains that carries a 125-bp insertion in the Por strain. b, Poolseq reads $(>150 \times$ coverage) of this position for Por and Jean, as shown in the integrated genome viewer (IGV). The reference genome does not have the 125-bp insertion. At the position marked by the red box, the Jean strain has a 4-bp polymorphic indel (ATAC, frequently misaligned due to a SNP 8 bp downstream), whereas the Por strain has the 125-bp insertion (but not the 4-bp ATAC insertion). In Jean all reads span the indel, suggesting that if the 125 -bp insertion is present in Jean at all, its frequency is very low. In contrast, in Por all reads but one end at this position, suggesting the frequency of the 125-bp insertion in Por is 154 of 155 reads or $>0.99$. 


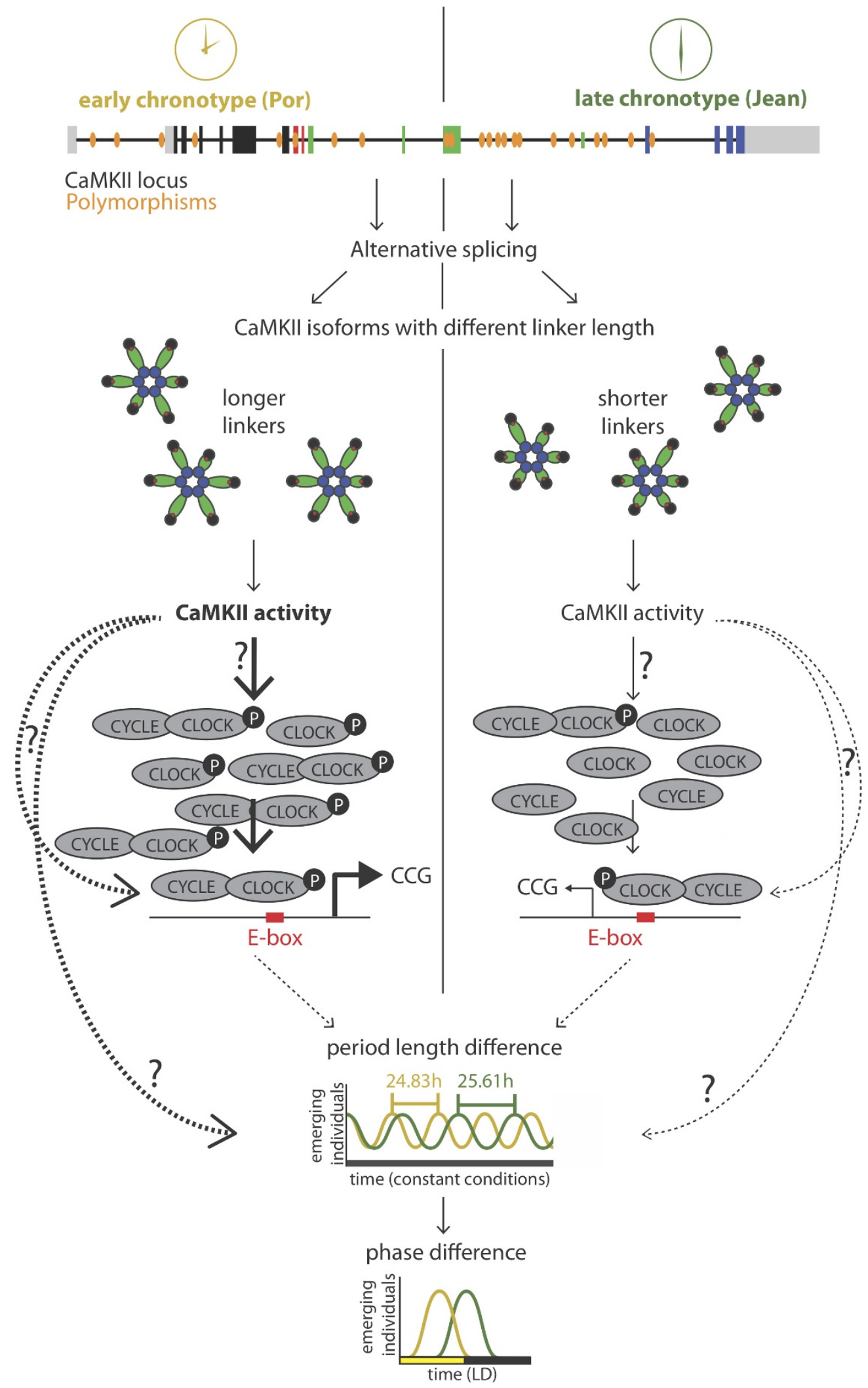

Extended Data Figure 8 Model of circadian timing adaptation via sequence differences in the CaMKII.1 genomic locus. Exon coloration as in Fig. 2b. The arrows with question marks indicate possible pathways that alone or in combination could mediate the effect of CaMKII.1 on timing. Dotted lines, indirect effects. 
a DNA

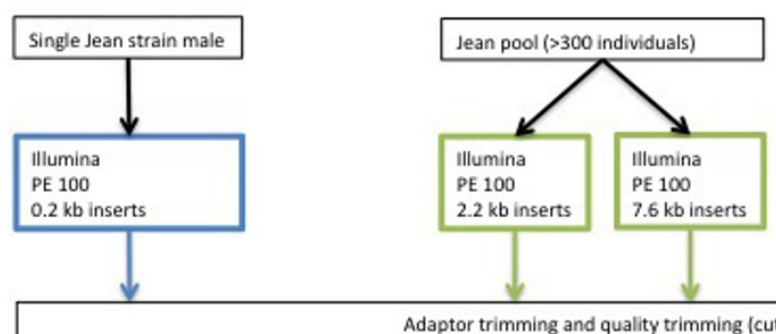

Mapping family. $54+2$ individuals

Sequencing

Read processing

Deduplication and further adaptor and quality trimming (EA-utils)
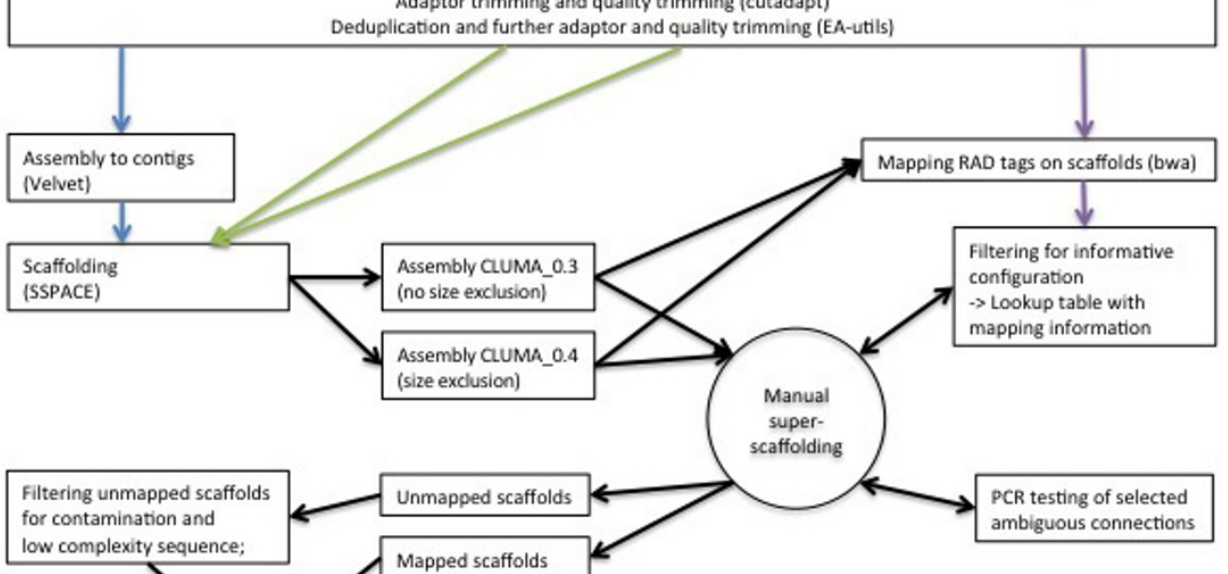

low complexity sequence;

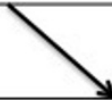

Closure of gaps (GapFiller)

Removal of repeated edges (custom script RE')

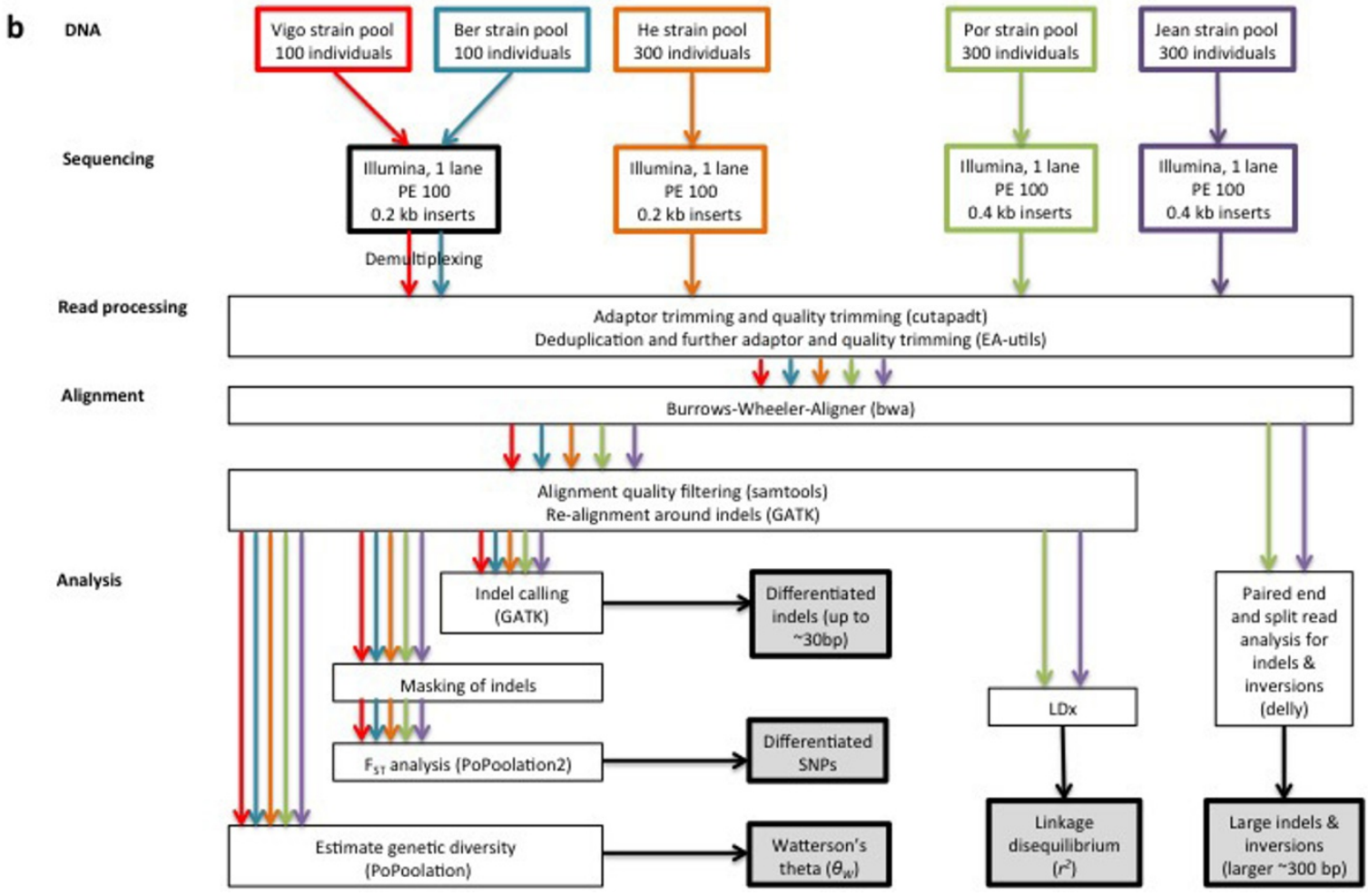

Extended Data Figure 9 | Analyses overview. a, Overview of the genome assembly process. b, Overview of the population genomic analyses. 
a

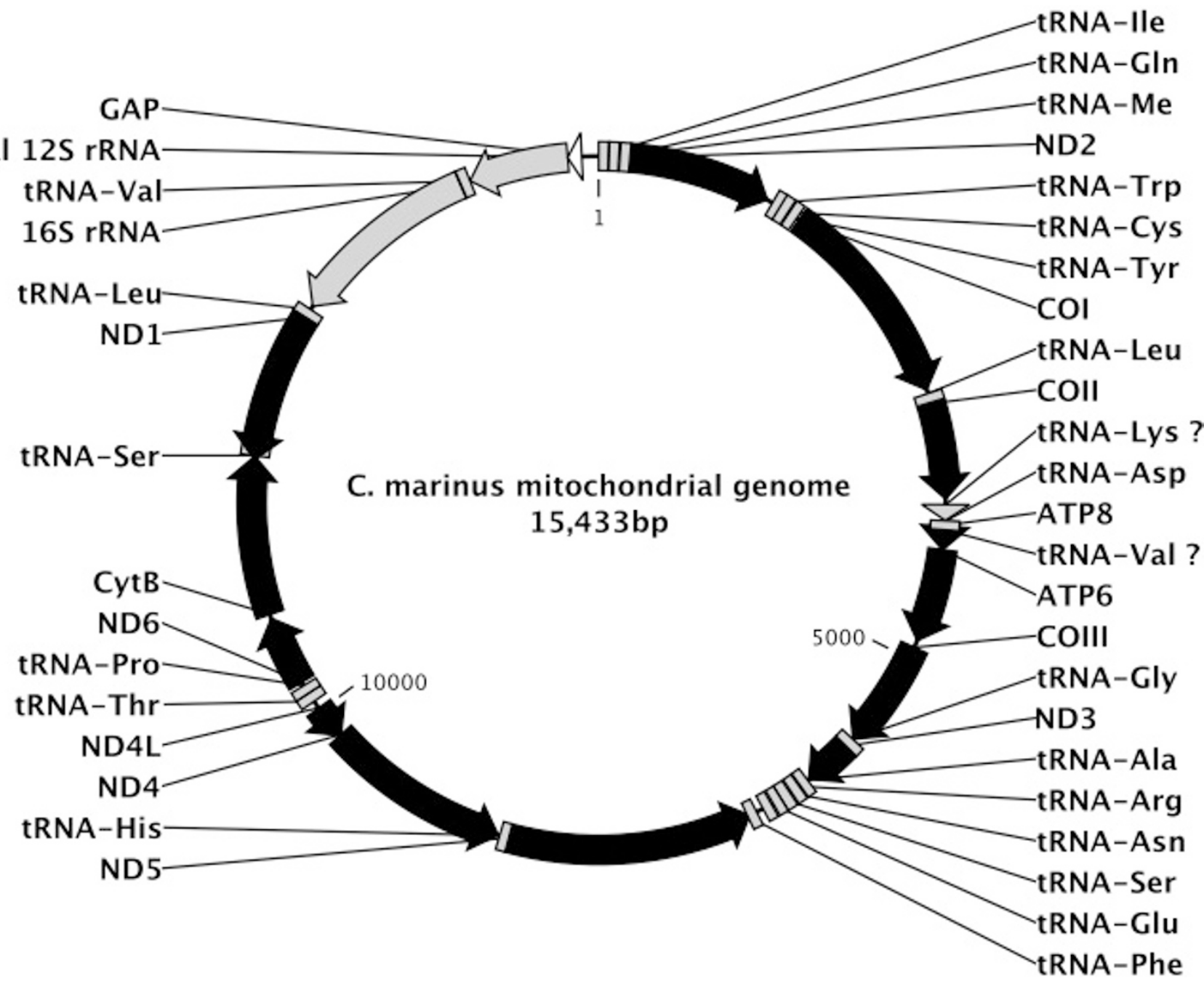

b

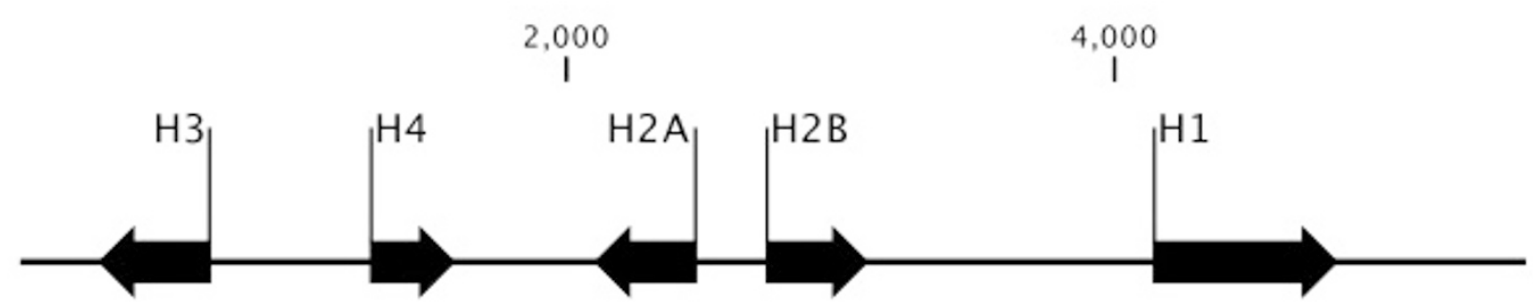

Extended Data Figure 10 | Arrangement of the mitochondrial genome and of the histone gene cluster in C. marinus. a, b, mitochondrial genome (a) and histone gene cluster (b) arrangements in C. marinus. Protein-coding genes are shown in black, tRNAs and rRNAs in grey. 